\title{
The asymptotic structure of a slender dragged viscous thread
}

\author{
MAURICE J. BLOUNT† AND JOHN R. LISTER \\ Institute of Theoretical Geophysics, Department of Applied Mathematics and Theoretical Physics, \\ University of Cambridge, Wilberforce Road, Cambridge CB3 0WA, UK \\ (Received 12 May 2010; revised 15 December 2010; accepted 30 December 2010; \\ first published online 23 March 2011)
}

The behaviour of a viscous thread as it falls onto a moving belt is analysed in the asymptotic limit of a slender thread. While the bending resistance of a slender thread is small, its effects are dynamically important near the contact point with the belt, where it changes the curvature and orientation of the thread. Steady flows are shown to fall into one of three distinct regimes, depending on whether the belt is moving faster than, slower than or close to the same speed as the free-fall velocity of the thread. The key dynamical balances in each regime are explained and the role of bending stresses is found to be qualitatively different. The asymptotic solutions exhibit the 'backward-facing heel' observed experimentally for low belt speeds, and provide the leading-order corrections to the stretching catenary in theory previously developed for high belt speeds. The asymptotic stability of the thread to the onset of meandering is also analysed. It is shown that the entire thread, rather than the bending boundary layer alone, governs the stability. A balance between the destabilising reaction forces near the belt and the restoring force of gravity on the remainder of the thread determines the onset of meandering, and an analytic estimate for the meandering frequency is thereby obtained. At leading order, neutral stability occurs with the belt moving a little more slowly than the free-fall velocity of the thread, not when the lower part of the thread begins to be under compression, but when the horizontal reaction force at the belt begins to be slightly against the direction of belt motion. The onset of meandering is the heel 'losing its balance'.

Key words: low-Reynolds-number flows

\section{Introduction}

The buckling of a viscous thread may be seen in several everyday situations, for example when pouring honey on toast or when squeezing toothpaste onto a toothbrush. The intriguing observation of steady coiling of a viscous thread as it falls onto a stationary surface has prompted many investigations. Early laboratory experiments (e.g. Barnes \& Woodcock 1958; Barnes \& MacKenzie 1959) made quantitative measurements of the dependence of coiling frequency on fall height and qualitative observations of the motion, but the physical processes governing the coiling were not explained.

An early theoretical advance was made by Taylor (1968), who drew an analogy between coiling viscous threads and buckling elastic beams, and concluded that coiling 
(a)

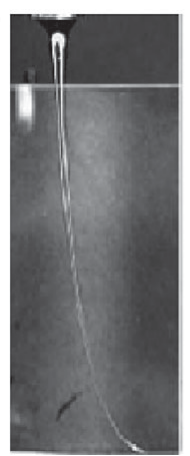

(b)

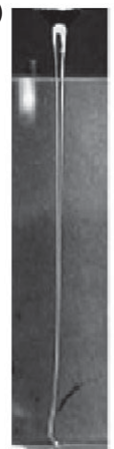

(c)

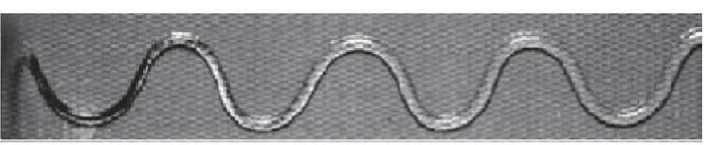

(d)

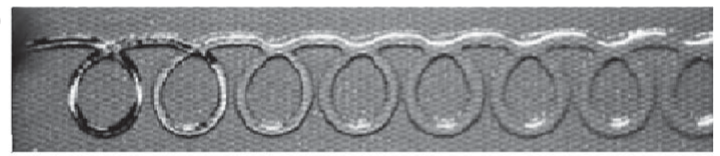

(e)

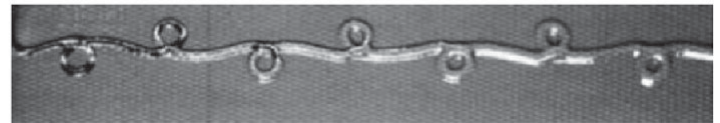

FIGURE 1. Experiments performed by Chiu-Webster \& Lister (2006), in which a viscous thread falls under gravity onto a belt moving from left to right. For slow belt speeds, the thread buckles and leaves behind a variety of patterns on the belt. $(a, b)$ Side views showing steady shapes at large and moderate belt speeds $\left(U_{B}=10.5 \mathrm{~cm} \mathrm{~s}^{-1}\right.$ and $7.5 \mathrm{~cm} \mathrm{~s}^{-1}$, with $H=10 \mathrm{~cm}$, $Q=0.025 \mathrm{~cm}^{3} \mathrm{~s}^{-1}, U_{N}=0.0314 \mathrm{~cm} \mathrm{~s}^{-1}$ and $\left.v=375 \mathrm{~cm}^{2} \mathrm{~s}^{-1}\right)$. (c-e) Top views showing some of the patterns $\left(Q=0.021 \mathrm{~cm}^{3} \mathrm{~s}^{-1}, U_{N}=0.0415 \mathrm{~cm} \mathrm{~s}^{-1}\right.$ and $\left.v \leqslant 390 \mathrm{~cm}^{2} \mathrm{~s}^{-1}\right)$. (c) Meandering with $U_{B}=3.8 \mathrm{~cm} \mathrm{~s}^{-1}$ and $H=8 \mathrm{~cm}$. (d) Translated coiling with $U_{B}=1.8 \mathrm{~cm} \mathrm{~s}^{-1}$ and $H=9 \mathrm{~cm}$. (e) Figures-of-eight with $U_{B}=4.4 \mathrm{~cm} \mathrm{~s}^{-1}$ and $H=11 \mathrm{~cm}$.

was an instability caused by axial compression of the thread. A quantitative model was developed by Entov \& Yarin (1984), who used a slender-thread approximation to determine the stability of fluid threads to transverse, long-wave disturbances. Mahadevan, Ryu \& Samuel $(1998,2000)$ analysed inertia-dominated coiling, in which regime bending stresses and centripetal forces balance within the coil. Their predictions of the scaling of the coiling frequency and radius gave good agreement with experiment.

Ribe (2004) presented a similar model to that of Entov \& Yarin (1984), but for the problem of steady coiling of a fluid thread. Ribe's model results in a 19th-order two-point boundary-value problem, and the coiling frequency predicted by his model agrees very well with experiment over a wide range of fall heights. The numerical solution also reveals the existence of three distinct regimes, which were confirmed experimentally by Maleki et al. (2006) and Habibi et al. (2006). Within these regimes, the bending stresses in the coil are either dominant, balanced by gravity, or balanced by inertia. The stability of the steady coiling solutions was investigated numerically by Ribe, Habibi \& Bonn (2006a). The conditions under which steady coiling is stable also agree with experimental observations. The coiling of a thread as it falls onto a stationary surface is therefore a problem that is fairly well understood.

The related problem of a viscous fluid thread falling onto a moving belt has only received similar attention more recently. A series of experiments were carried out by Chiu-Webster \& Lister (2006, hereafter CWL) using golden syrup with kinematic viscosity $>10^{2} \mathrm{~cm}^{2} \mathrm{~s}^{-1}$ falling through typical heights of $3-13 \mathrm{~cm}$ onto a belt moving at typical speeds of $1-15 \mathrm{~cm} \mathrm{~s}^{-1}$. CWL discovered that, while at higher belt speeds such a viscous thread forms a steady shape similar to a catenary (figure 1a), at lower belt speeds the buckling of the thread and the motion of the belt combine to create a fascinating variety of patterns as the thread falls on the belt (figure $1 c-e$ ). These patterns include 'meanders' (figure 1c) across the belt and 'translated coils' (figure $1 d$ ) in addition to other more exotic patterns such as 'figures-of-eight' (figure 1e), 'braiding' and 'slanted loops'. The critical belt speed between the 
steady and the unsteady behaviour increased roughly quadratically with the fall height.

CWL described a simple theory for the steady catenary shapes, in which the bending resistance of the thread is neglected, and the thread is governed by a dominant balance between axial stretching and gravity. The effects of inertia and surface tension are included, but are much smaller than the extensional viscous stresses for the experimental parameters considered. Similar equations for viscous jets in the absence of bending stresses have been described in the context of fibre pulling and rotary spinning (e.g Dewynne, Ockendon \& Wilmott 1992; Dewynne, Howell \& Wilmott 1994; Cummings \& Howell 1999; Decent et al. 2009; Marheineke $\&$ Wegener 2009). The predicted shape of the steady catenaries agreed well with the experimental observations for large belt speeds, but the neglect of bending stresses causes the theory to break down at lower belt speeds when the bottom of the thread ceases to be under tension. However, steady thread shapes are observed at some of these lower belt speeds when no steady stretching catenary exists. The threads still meet the belt tangentially, and so these steady shapes cannot simply be described as vertical stagnation-flow-like solutions. Moreover, the 'backward-facing heel' of shapes observed at these lower speeds near the onset of unsteady motion (figure $1 b$ ) cannot be explained without bending stresses.

The fall of a fluid thread onto a moving belt has also been considered theoretically and experimentally by Hlod et al. (2007) and Hlod (2009), with an emphasis on larger velocities than CWL so that the inertial momentum flux in the thread is important. By neglecting surface tension, Hlod et al. (2007) were able to make further analytic progress and prove a number of results about solutions to fluid-jet equations with inertia, extensional stress and gravity. In particular, they showed, as did Dyson (2007), that when the momentum flux dominates the viscous stress the direction of the characteristics makes it appropriate to impose tangency at the nozzle rather than at the belt. (Ballistic trajectories can thus be obtained with angled nozzles.) Bending stresses are neglected and these boundary conditions for high-speed jets should not be confused with those applicable to the low-speed viscous threads in this paper for which bending stresses are dominant at the boundaries. Dyson (2007) also analysed the fall of a fluid sheet onto a moving belt in the context of curtain coating in the paper industry. The focus is on inertia-dominated fall, but there is some analysis of the effects of bending stresses in sheets, which parallels some of the analysis of viscous threads here. Clearly, a sheet cannot meander or form patterns in the same way as a thread.

The stability of a steadily dragged thread to transverse disturbances was analysed by Ribe, Lister \& Chiu-Webster (2006b). Their analysis includes bending stresses in both the unperturbed and perturbation equations, and the predicted steady shapes agree very well with those observed experimentally. The onset and frequency of meandering predicted by the model also correspond closely to experiment. The mathematical model includes many dynamical effects and results in a complicated 17th-order twopoint boundary-value problem, which makes it a little difficult to extract a more physically based understanding of the solution structure and instability.

Morris et al. (2008) significantly improved the experimental method of CWL and gave an extensive cartography of the unsteady behaviours observed in experiments with silicon oil (kinematic viscosity $277 \mathrm{~cm}^{2} \mathrm{~s}^{-1}$ ) over a range of fall heights and belt speeds that are a little smaller than those of CWL. They also used a phenomenological theory to estimate the amplitude of meanders near the onset of instability. An attempt to set the experimental observations in the framework of weakly nonlinear amplitude 
equations and bifurcation theory was only partly successful, perhaps because a simple modal description does not capture the dynamical structure that we explore in this paper.

Our aim is to provide analytical and physical understanding of some observations of CWL and Morris et al. (2008), in the regime where inertial effects are negligible. Our work describes the effects of bending stress on a very viscous thread, that is falling and being strongly stretched by gravity, in the asymptotic limit of a very slender thread where the thread radius is much smaller than the nozzle height. Particular attention is paid to the influence of bending stresses near the belt. These bending stresses give rise to singular perturbations to the theory of CWL in which they are omitted and, in consequence, there are boundary layers near the belt and the nozzle within which the bending stresses are dynamically important. A significant part of this paper focuses on elucidating the boundary-layer structure and its effects.

The dynamics of the thread falls into one of three regimes depending on the relative sizes of $U_{B}$ and a 'free-fall' speed $U_{F}$ which we will determine. The first regime, in which $U_{B}<U_{F}$, predicts the observed backward-facing heels that could not be explained by previous work that omitted bending stress. The regime $U_{B}>U_{F}$ gives small corrections to such theories. The third regime, $U_{B} \approx U_{F}$, is distinct and bridges the other two regimes. In each of the above cases, we determine the influence of bending stresses, derive scalings and physical balances within the boundary layer, and find asymptotic approximations that agree well with the full numerical model of Ribe et al. (2006b).

In the second part of the paper, we use our new understanding of the steady solutions to derive an asymptotic estimate of the onset of instability to meandering oscillations. This estimate is significantly better than that of CWL, which approximated the onset of instability by the loss of catenary solutions, which corresponds to the assumption that the thread is unable to support any compression. In contrast, our analysis demonstrates that bending stresses near the belt can support a limited amount of compression and thus the onset is at lower belt speeds, as observed. We also find that the pinning of the thread at the nozzle plays an important role since the frequency of meandering oscillations is governed by a balance between bending stresses near the belt and the restoring force exerted by the nozzle. We use our understanding of this mechanism to give a simple estimate of the frequency of meandering near onset, and to determine the structure of the eigenmode.

\section{Problem description}

We consider a viscous thread falling a distance $H$ from a nozzle onto a belt moving horizontally with constant speed $U_{B}$, as depicted in figure 2 . The thread has dynamic viscosity $\mu$ and density $\rho$. The volume flux $Q$ from the nozzle is constant, and the extrusion speed is $U_{N}$. We assume for the moment that the thread is steady. We also assume that the radius $a$ of the thread is much smaller than $H$ throughout its length. This allows the thread to be modelled by its curved centreline, parametrised by the arclength $s$ measured backward from the contact point, and whose dynamical properties are derived by taking averages over the cross-section of the thread.

We denote the position of the thread's centreline by $\boldsymbol{x}(s)$, where $\boldsymbol{x}=(x, y, z)$ with respect to fixed Cartesian axes chosen so that the thread lies in the plane $y=0$, the belt is at $z=0$ and the nozzle is at $(0,0, H)$. The belt moves with velocity $U_{B} \boldsymbol{e}_{x}$ and the gravitational acceleration is $-g \boldsymbol{e}_{z}$. The orientation of the thread is given by $\theta(s)$, defined as the angle between the centreline and the downward vertical. It is convenient 


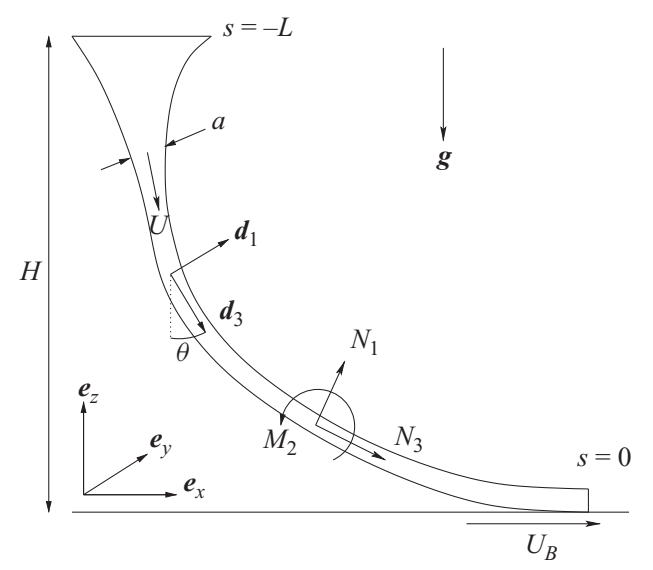

FIGURE 2. Definition of the problem (see text).

to define a Lagrangian triad of basis vectors $\boldsymbol{d}_{i}(s)$ which rotate with the thread as it flows. We choose $\boldsymbol{d}_{3}$ to be tangential to the thread, and to have the same sense as the fluid velocity so that $\boldsymbol{d}_{3} \cdot \boldsymbol{e}_{z}=-\cos \theta$. The vectors $\boldsymbol{d}_{1}$ and $\boldsymbol{d}_{2}$ may rotate within the cross-section of the thread. However, when the thread is steady, it remains in the $y=0$ plane and does not twist. Hence, we can choose $\boldsymbol{d}_{2}=-\boldsymbol{e}_{y}$ throughout the thread. The right-handed triad is then completed by $\boldsymbol{d}_{1}=\boldsymbol{d}_{2} \times \boldsymbol{d}_{3}$, which is normal to and in the plane of the centreline. The variation of the basis vectors along the centreline is governed by the curvature $\boldsymbol{\kappa}$ of the thread according to $\boldsymbol{d}_{i}^{\prime}=\boldsymbol{\kappa} \times \boldsymbol{d}_{i}$, where a prime denotes $\mathrm{d} / \mathrm{d} s$. Since $y=0$ throughout the thread, the only non-zero component of curvature is $\kappa_{2}=\boldsymbol{\kappa} \cdot \boldsymbol{d}_{2}$. The geometry of the centreline is thus described by

$$
\begin{aligned}
x^{\prime} & =\sin \theta, \\
z^{\prime} & =-\cos \theta, \\
\theta^{\prime} & =\kappa_{2},
\end{aligned}
$$

where primes again denote $\mathrm{d} / \mathrm{d} s$. Since the thread is slender, we may assume that the axial velocity $U \boldsymbol{d}_{3}$ is uniform across a cross-section at leading order and that the cross-sections are approximately circular. Hence, $Q=\pi a^{2} U$.

We now consider the dynamics of the thread. For simplicity of exposition, and in order to focus on understanding the effects of viscous bending stresses, we neglect the effects of surface tension and inertia, which CWL showed - see the terms representing inertia and surface tension in their dimensionless equation (4.9) - is appropriate when the dimensionless parameters

$$
R e=\left(\frac{U_{B}^{3}}{3 v g}\right)^{1 / 2} \quad \text { and } \quad \Gamma=\frac{\gamma}{\rho}\left(\frac{\pi}{3 v g Q}\right)^{1 / 2}
$$

are both small. The range of $R e$ and $\Gamma$ was $10^{-3}$ to $10^{-1}$ and $0.4-0.7$ in the experiments of CWL and $10^{-3}$ to $4 \times 10^{-2}$ and 0.26 in the experiments of Morris et al. (2008). The neglect of surface tension is thus a slightly crude approximation for quantitative predictions, but it does not change the structure of the asymptotic analysis and solutions. In Appendix A, we explain how our analysis may straightforwardly be modified to re-include the effects of surface tension, the main effect being a modification of the rate of stretching between the nozzle and the belt. In the Appendix, 
we also describe the circumstances under which the effects of inertia may similarly be included.

The dynamics of the thread involves the stress vector $N(s)$ and stress-moment vector $\boldsymbol{M}(s)$ acting on a cross-section, which are given by

$$
\begin{gathered}
N(s)=N_{i} \boldsymbol{d}_{i}=\int \boldsymbol{\sigma}(\boldsymbol{x}+\boldsymbol{y}) \cdot \boldsymbol{d}_{3} \mathrm{~d} A_{\boldsymbol{y}}, \\
\boldsymbol{M}(s)=M_{i} \boldsymbol{d}_{i}=\int \boldsymbol{y} \times \boldsymbol{\sigma}(\boldsymbol{x}+\boldsymbol{y}) \cdot \boldsymbol{d}_{3} \mathrm{~d} A_{\boldsymbol{y}},
\end{gathered}
$$

where $\boldsymbol{\sigma}$ is the stress tensor, $\boldsymbol{y}$ denotes the displacement from the centreline within the cross-section and the integral is taken over the cross-section.

The axial stress $N_{3}$ is due to extensional flow, and given by the Trouton relation

$$
N_{3}=3 \pi a^{2} \mu U^{\prime}
$$

The bending stress moment $M_{2}$ may be obtained through expansion of the velocity profile in the thread to second order in $\boldsymbol{y}$, finding the stress tensor and then substituting it into the above expression for $\boldsymbol{M}$. This yields (Entov \& Yarin 1984; Yarin 1993)

$$
M_{2}=\frac{3 \pi a^{4} \mu}{8}\left(2 U \kappa_{2}^{\prime}-U^{\prime} \kappa_{2}\right)
$$

We note that the derivation of the stress moment in Ribe et al. (2006a) does not consider the coupling between bending and stretching and thus omits the second term of (2.8). However, as we show in $\S 3.2, U^{\prime} \kappa_{2} \ll U \kappa_{2}^{\prime}$ in the bending boundary layers and so the absence of this term is of little consequence. We also note that the analogous expression for the stress moment in a bending fluid sheet (e.g. Buckmaster, Nachman \& Ting 1978; Griffiths \& Howell 2007) is slightly different, in part because there are circulatory flows within the cross-section of a bending thread which cannot occur by mass conservation in a two-dimensional sheet.

Force balances in the directions normal and tangential to the thread imply that

$$
\begin{aligned}
& N_{1}^{\prime}=-\kappa_{2} N_{3}+\pi a^{2} \rho g \sin \theta, \\
& N_{3}^{\prime}=\kappa_{2} N_{1}-\pi a^{2} \rho g \cos \theta .
\end{aligned}
$$

The balance of moments implies that

$$
M_{2}^{\prime}=-N_{1}-\frac{\pi a^{4}}{4} \rho g \kappa_{2} \cos \theta .
$$

The appropriate boundary conditions on the viscous thread at the nozzle are analogous to the 'clamped' conditions that are often applied in the context of static deformations of elastic threads and sheets. The thread at the nozzle is assumed to be aligned with the nozzle (so that it is vertical) and to have zero curvature. From (2.3) and (2.8), such conditions are necessary to avoid introducing a point couple at the nozzle. Similarly, the average velocity must be continuous to avoid introducing a point force at the nozzle. Hence, we impose

$$
\theta=\kappa_{2}=x=0, \quad z=H \quad \text { and } \quad U=U_{N},
$$

at the nozzle. The adjustment of the velocity profile from Poiseuille flow inside the nozzle to an extensional flow in the thread takes place over a length scale comparable with the thread's radius (Goren \& Wronski 1966) and has an asymptotically negligible effect in the limit of a slender thread. 
If the fall height or the belt speed were large enough that inertial effects were dominant near the bottom of the thread, then the impact of the thread on the belt might result in a stagnation-point flow. However, in this paper we focus on the regime where viscous effects are dominant. The appropriate boundary conditions for such a viscous thread are rolling conditions at the contact point (Ribe 2004; Ribe et al. $2006 \mathrm{~b}$ ), which require both the angle and curvature of the thread to be continuous with the fluid at the belt in order to ensure that there is no point couple at the contact. Similarly, we avoid introducing a point force at the contact point by imposing the condition that the velocity is continuous. We make the approximation that the height of the centreline at the contact point is at $z=0$; the actual height is equal to the thread's radius, but the difference is negligible in the asymptotic limit of a very slender thread. In summary, we impose the conditions

$$
\theta=\frac{\pi}{2}, \quad \kappa_{2}=0, \quad z=0 \quad \text { and } \quad U=U_{B}
$$

at the belt.

The thread is described by the eight independent variables $x, z, \theta, \kappa_{2}, U, N_{1}, N_{3}$ and $M_{2}$. The radius of the thread is determined by the velocity through flux conservation, which implies that $\pi a^{2} U=Q$. In addition to the eight independent variables, the length $L$ of the thread is unknown. Hence, the nine boundary conditions (2.12) fully determine the solution. We note that these boundary conditions are directly analogous to those used in the analysis of coiling and they give solutions that are in excellent agreement with experimental observations both of coiling (Habibi et al. 2006) and of a dragged thread (Ribe et al. 2006b).

The solution to the system of equations (2.1)-(2.12) may be computed using the continuation software package AUTO (see Ribe et al. 2006a; Doedel \& Oldeman 2009), which is freely available at http://indy.cs.concordia.ca/auto/. A continuation method has the advantage that solutions are easily computed when experimental variables, such as $U_{B}$ or $H$, are varied.

It is known (CWL; Ribe et al. 2006b) that the bending resistance of the thread becomes dynamically important near the belt, but the significance of this has not been quantitatively explored. We aim to analyse the behaviour of the thread in this region, and hence we scale our equations with respect to the corresponding dynamical balances.

We non-dimensionalise the velocity with $U_{E}=\rho g H^{2} / \mu$, which is an extensional velocity scale associated with a thread as it falls and stretches under a viscousgravity balance. (We find the vertical variation of velocity for such a thread in $\S 3.1$.) The thread radius is non-dimensionalised with the corresponding radial scale $a_{E}=\sqrt{Q / \pi U_{E}}$, and axial lengths with $H$. The constitutive relation (2.7) and the stress-moment balance (2.11) suggest that the stress components $N_{i}$ should be nondimensionalised with $\mu a_{E}^{2} U_{E} / H$ and the stress moments $M_{i}$ with $\mu a_{E}^{2} U_{E}$. In the new dimensionless variables, (2.1)-(2.11) become

$$
\begin{aligned}
x^{\prime} & =\sin \theta, \\
z^{\prime} & =-\cos \theta, \\
\kappa_{2} & =\theta^{\prime}, \\
N_{3} & =3 \pi a^{2} U^{\prime}, \\
M_{2} & =\frac{3 \pi a^{4}}{8} \epsilon^{2}\left(2 U \kappa_{2}^{\prime}-U^{\prime} \kappa_{2}\right),
\end{aligned}
$$




$$
\begin{aligned}
& N_{1}^{\prime}=-\kappa_{2} N_{3}+\pi a^{2} \sin \theta, \\
& N_{3}^{\prime}=\kappa_{2} N_{1}-\pi a^{2} \cos \theta, \\
& M_{2}^{\prime}=-N_{1}-\frac{\pi a^{4}}{4} \epsilon^{2} \kappa_{2} \cos \theta,
\end{aligned}
$$

where $a^{2} U=1$. The parameter

$$
\epsilon=\frac{a_{E}}{H}=\left(\frac{Q \mu}{\pi \rho g H^{4}}\right)^{1 / 2}
$$

is the slenderness of the thread, and the analysis of this paper concerns the asymptotic behaviour as $\epsilon \rightarrow 0$. The non-dimensionalisation of the variables modifies the boundary conditions $(2.12 d, e, i)$ to

$$
U=U_{n}, \quad z=1 \quad \text { at } \quad s=-\ell \text { and } U=U_{b} \quad \text { at } \quad s=0,
$$

where $\ell=L / H$ is the dimensionless length of the thread, and $U_{n}=U_{N} / U_{E}$ and $U_{b}=U_{B} / U_{E}$ are the dimensionless nozzle velocity and belt speed, respectively. The analysis that follows also involves the dimensionless thread radius $a_{b}=U_{b}^{-1 / 2}$ at the belt and the 'free-fall' speed $U_{f}=U_{F} / U_{E}$.

Throughout this paper we make the further assumption that before reaching the belt the thread undergoes strong stretching by gravity, so that $U_{n} \ll 1$. This stretching is necessary for there to be a separation between the length scale across which bending stresses are dynamically important and the unit dimensionless height. Values of $U_{n}$ in the experiments of CWL and Morris et al. (2008) range from $10^{-3}$ to $10^{-6}$. If flow through the nozzle is gravity-driven, then $U_{n} \propto \epsilon$ and strong stretching is implied by slenderness.

\section{Asymptotic behaviour of a very slender thread}

\subsection{The outer solution or 'tail'}

CWL analysed the behaviour of a slender dragged thread by assuming that the thread has a negligible bending resistance; this corresponds to setting $\epsilon=0$ in (2.17) and (2.20). Then, $M_{2}=N_{1}=0$ and the thread is governed by

$$
\begin{aligned}
x^{\prime} & =\sin \theta, \\
z^{\prime} & =-\cos \theta, \\
N_{3} & =3 \pi a^{2} U^{\prime}, \\
N_{3} \theta^{\prime} & =\pi a^{2} \sin \theta, \\
N_{3}^{\prime} & =-\pi a^{2} \cos \theta .
\end{aligned}
$$

CWL found that catenary-like solutions, for which the thread is tangential to the belt at contact, only exist if the belt speed $U_{b}$ is larger than some critical value, which we denote $U_{f}$. For $U_{b}>U_{f}$, the belt exerts a horizontal force on the thread placing the bottom of it under axial tension, so that $N_{3}>0$ throughout the thread and the global behaviour of the thread is to hang in a catenary shape.

If $U_{b}<U_{f}$, there are no steady catenary solutions to (3.1)-(3.5). However, there are solutions in which the thread falls vertically, with $\theta=0$ everywhere. Substitution of $\theta=0$ into (3.1)-(3.5) gives $x=0, z=-s$ and

$$
\left(3 \pi a^{2} U^{\prime}\right)^{\prime}+\pi a^{2}=0 .
$$


Using $a^{2}=1 / U$, it is easy to show that the general solution to (3.6) is

$$
U(s)=\frac{1-\cos \left[T_{\infty}(s+1+d)\right]}{3 T_{\infty}^{2}},
$$

where $T_{\infty}$ and $d$ are constants of integration. An equivalent solution is given in Ribe (2004). As explained in $\S 2$, we consider only the case of strongly stretched threads for which $U_{n} \ll 1$. The nozzle condition $U=U_{n}$ at $s=-1$ gives

$$
d=\sqrt{6 U_{n}}+O\left(U_{n} T_{\infty}^{2}\right)
$$

(CWL noted that $d$ corresponds to the small offset above the nozzle where the thread speed would vanish if the solution were continued upwards in $s<-1$.) The remaining constant $T_{\infty}$ can be chosen to satisfy $U=U_{b}$ at $s=0$, though the resulting solution clearly does not satisfy the rolling conditions $(2.12 f, g)$.

If $U_{b}<U_{f}$, the bottom of this vertical fall solution satisfies $U^{\prime}(0)<0$ and the fall of the thread is slowed by means of a reaction force $-N_{3}(0)$ exerted upwards by the belt. Conversely, if $U_{b}>U_{f}$ then $U^{\prime}(0)>0$ and $N_{3}(0)>0$, and the fall speed must be increased by applying a downward force at the bottom of the thread. While the latter case may be relevant to pulling of polymer or glass fibres (e.g. Matovich \& Pearson 1969; Dewynne, Ockendon \& Wilmott 1989), it cannot be relevant to a thread simply falling on a belt where the reaction force must clearly be upwards. Hence, vertical-fall solutions are only relevant here when $N_{3}(0)<0$.

The transition between vertical-fall solutions for $N_{3}(0)<0$ and the catenary-like solutions for $N_{3}(0)>0$ occurs when $N_{3}(0)=0$; for this solution the bottom of the thread is stress-free and has a speed $U_{f}$ that we thus refer to from now on as the 'free-fall' speed. (This use of free-fall speed should not be confused with the idea of free-fall in mechanics for a pure inertia-gravity balance.) The free-fall speed is determined by applying the stress-free condition $U^{\prime}=0$ at $s=0$ to the general solution (3.7). We obtain $T_{\infty}=\pi /(1+d)$ and thus

$$
U_{f}=\frac{2}{3 \pi^{2}}+O(d)
$$

Because of its transitional nature, $U_{f}$ is a key parameter for the solution structure.

The system (3.1)-(3.5) no longer involves the derivatives of $\kappa_{2}, M_{2}$ and $N_{1}$ and so its order is three fewer than (2.13)-(2.20). The vertical-fall equations no longer involve the derivative of $\theta$ and their order is one fewer still. Consequently, both 'vertical fall' and 'catenary' solutions do not satisfy all of the orientation and curvature conditions at the belt and at the nozzle. It follows that the finite bending resistance of the thread for $0<\epsilon \ll 1$ gives rise to a singular perturbation to the system (3.1)-(3.5) where bending stress is neglected. This singular perturbation causes there to be boundary layers at the belt and at the nozzle, across which the curvature and orientation of the thread are corrected to satisfy the relevant boundary conditions. Outside these boundary layers, in a region we will, following Ribe, from now on refer to as the 'tail', bending stresses are unimportant and the solution can be approximated by a vertical-fall or catenary solution.

\subsection{Boundary-layer structure}

In the following subsections, we determine the boundary-layer structure produced by the singular perturbation of (3.1)-(3.5) by the small bending resistance of a slender thread. A preliminary simplification to note is that the $O\left(\epsilon^{2}\right)$ gravitational 
contribution to the moment balance $(2.20)$ is only a small regular perturbation as $\epsilon \rightarrow 0$, and can be omitted.

The bending stresses in the thread decrease as $\epsilon \rightarrow 0$, but not uniformly, and they remain dynamically important in boundary-layer regions of decreasing length near the belt and the nozzle. We focus our attention on the boundary layer near the belt, whose length scale we denote by $\delta_{*}$. The tail is governed by (3.1)-(3.5), and is therefore independent of $\epsilon$ at leading order. Hence, the axial stress, velocity and non-dimensionalised radius at the base of the thread are independent of $\epsilon$, and remain $O(1)$ as $\epsilon \rightarrow 0$. The constitutive relation (2.16) then implies that $U^{\prime}=O(1)$ in the boundary layer, and hence that $U$ cannot vary significantly over the $O\left(\delta_{*}\right)$ length scale of the boundary layer. The necessary adjustment of the thread velocity from its free-fall speed $U_{f}$ to the belt speed $U_{b}$ must therefore take place within the tail. Hence, we can make the leading-order approximation that $U=U_{b}$ throughout the boundary layer at the belt.

The constitutive relation (2.17) for the stress moment $M_{2}$ may also be simplified in the bending boundary layer. If the curvature in the boundary layer scales like $\kappa_{*}$ then, to satisfy $(2.12 g), \kappa_{2}^{\prime}$ must be $O\left(\kappa_{*} / \delta_{*}\right)$. Hence, the term $U \kappa_{2}^{\prime}$ in $(2.17)$ is $O\left(U_{b} \kappa_{*} / \delta_{*}\right)$. In contrast, since $U^{\prime}=O(1)$, the term $U^{\prime} \kappa_{2}$ is only $O\left(U_{b} \kappa_{*}\right)$, and is therefore negligible. This justifies omission of the second term, and henceforth we substitute $a_{b}^{2} U_{b}=1$ to obtain

$$
M_{2}=\frac{3 \pi a_{b}^{2}}{4} \epsilon^{2} \kappa_{2}^{\prime}
$$

We combine (3.10) with (2.18)-(2.20) to obtain the simplified fifth-order system of dynamic equations

$$
\begin{aligned}
& N_{1}=-\frac{3 \pi a_{b}^{2}}{4} \epsilon^{2} \theta^{\prime \prime \prime}, \\
& N_{1}^{\prime}=-\kappa_{2} N_{3}+\pi a_{b}^{2} \sin \theta, \\
& N_{3}^{\prime}=\kappa_{2} N_{1}-\pi a_{b}^{2} \cos \theta .
\end{aligned}
$$

We note that the force balances (3.12) and (3.13) have first integrals $F_{x}$ and $F_{z}$, which are the horizontal and vertical forces exerted by the belt, given by

$$
F_{x}=N_{3} \sin \theta+N_{1} \cos \theta \text { and } F_{z}=N_{1} \sin \theta-N_{3} \cos \theta-\pi a_{b}^{2} s,
$$

respectively. Hence, (3.11)-(3.14) may be recast as the third-order system,

$$
\begin{aligned}
\epsilon^{2} \theta^{\prime \prime \prime} & =-\frac{4}{3 \pi a_{b}^{2}} N_{1}, \\
N_{1} & =F_{x} \cos \theta+F_{z} \sin \theta+\pi a_{b}^{2} s \sin \theta,
\end{aligned}
$$

where $F_{z}$ and $F_{x}$ are as yet unknown but are determined by matching to the tail solution. The axial stress $N_{3}$ is

$$
N_{3}=F_{x} \sin \theta-F_{z} \cos \theta-\pi a_{b}^{2} s \cos \theta .
$$

The qualitative nature of the boundary-layer corrections depends on the relative sizes of the free-fall speed $U_{f}$ and the belt speed $U_{b}$. The variation of the velocity in the tail requires a force to be exerted through the boundary layer. If $U_{b}<U_{f}$, the boundary layer exerts a vertical force $F_{z}$ on the thread, which reduces the stretching and slows the fall of the tail above it. The tail is nearly vertical with velocity variation given approximately by the solution (3.7). If $U_{b}>U_{f}$, the boundary layer exerts a horizontal force $F_{x}$ on the tail, which increases the stretching and accelerates the 
thread. The thread is deflected sideways and the axial velocity variation within the tail is given approximately by that of the catenary-like solution to (3.1)-(3.5). There is also an intermediate regime, where $U_{b} \approx U_{f}$, in which $F_{x}$ and $F_{z}$ are of similar size in the boundary layer.

Within each regime, there are distinct dynamical balances in the boundary layer. We will introduce scaled variables

$$
\eta=\frac{s}{\delta_{*}} \quad \text { and } \quad n_{i}=\frac{N_{i}}{F_{*}},
$$

where $\delta_{*}$ and $F_{*}$ are respectively the length and force scales that are relevant to each regime under consideration.

\subsection{Compressional heel: $U_{b}<U_{f}$}

We first deal with the case in which the belt speed $U_{b}$ is slower than the free-fall velocity $U_{f}$. The necessary decrease in fall speed within the tail places the lower part of it under compression. The role of bending stresses within the boundary layer at the belt is to support this compression, and to divert the thread from $\theta \approx 0$ in the tail to $\theta=\pi / 2$ at the belt.

The compressive force $F_{z}$ required to slow the fall of the tail is $O(1)$ as $\epsilon \rightarrow 0$. The force balance $(3.14 b)$ implies that the contribution of the gravitational stress to $F_{z}$ within the boundary layer is $O\left(\delta_{z}\right)$, where $\delta_{z}$ is the length scale of the boundary layer, and thus negligible as $\epsilon \rightarrow 0$. Furthermore, we anticipate that both $N_{1} \rightarrow 0$ and $\theta \rightarrow 0$ in the tail and so (3.14a) motivates the further assumption that $F_{z} \gg F_{x}$. Hence, $N_{1} \sim N_{3} \sim F_{z}$ within the boundary layer. The change in $\theta$ from 0 to $\pi / 2$ across the boundary layer implies that the curvature $\kappa_{2}=\theta^{\prime} \sim \delta_{z}^{-1}$. We substitute these scalings into the stress-moment equation (3.15) to obtain

$$
\delta_{z}=\left(\frac{3 \pi a_{b}^{2} \epsilon^{2}}{4 F_{z}}\right)^{1 / 3}
$$

We note that $\delta_{z}$ is $O\left(\epsilon^{2 / 3}\right)$, and hence our earlier neglect of the $O(\epsilon)$ corrections (such as from the radius of the thread at the contact point) is justified.

Using $F_{z}$ and $\delta_{z}$ to define the rescaling (3.18), we find that the bending stress (3.16) is given at leading order by

$$
n_{1}=\sin \theta \text {. }
$$

The rescaled shape $\theta(\eta)$ of the thread is obtained by substitution into (3.15) to obtain

$$
\theta^{\prime \prime \prime}+\sin \theta=0 .
$$

The boundary conditions at the belt are

$$
\theta=\frac{\pi}{2} \quad \text { and } \quad \theta^{\prime}=0 \quad \text { at } \quad \eta=0
$$

A third condition is required to match to a vertical tail and enforce the decay of bending stress. We anticipate that $\theta \approx 0$, and linearisation about this value implies that

$$
\theta \sim \sum_{i=1,2,3} A_{i} \exp \left(\lambda_{i} \eta\right) \quad \text { as } \quad \eta \rightarrow-\infty,
$$

where $\lambda_{i}^{3}=-1$ and $A_{i}$ are complex amplitudes of the three modes. Two of these modes decay exponentially as $\eta \rightarrow-\infty$ as desired, but the mode with $\lambda_{i}=-1$ diverges. 


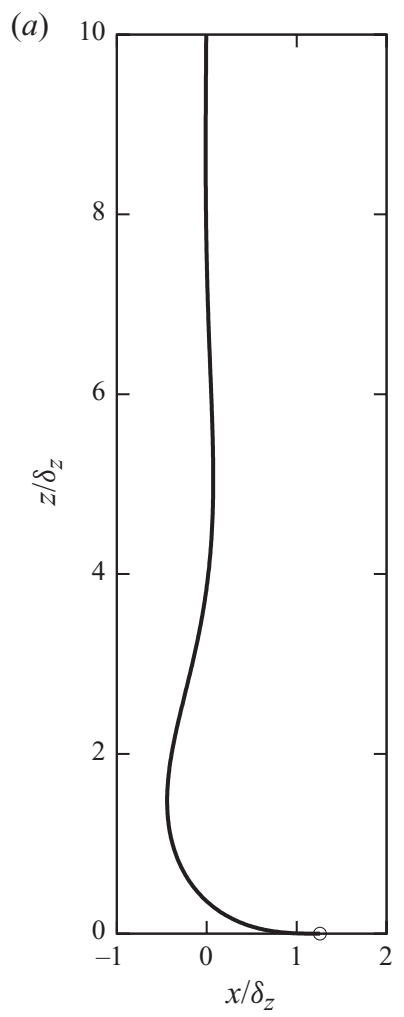

(b)

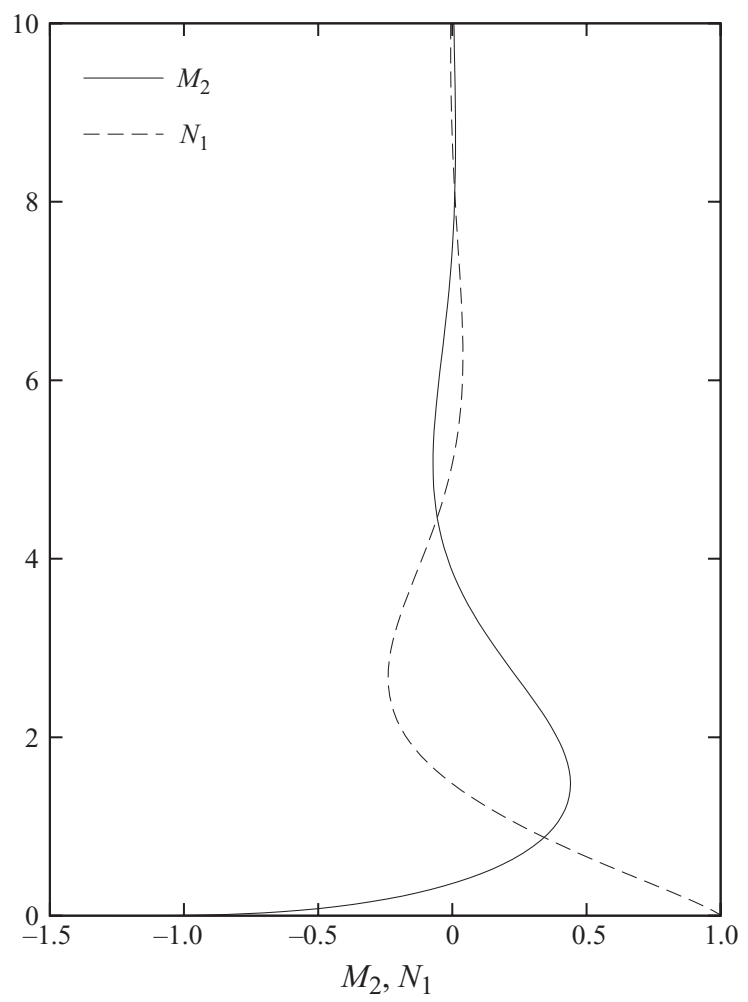

Figure 3. (a) The universal shape of the compressional-heel solution to (3.21), (3.22) and (3.24). (b) The distributions of the stress moment $M_{2}$ (solid) and the bending stress $N_{1}$ (dashed) in the compressional heel.

Thus, suppression of this divergent mode by imposing

$$
\theta \rightarrow 0 \quad \text { as } \quad \eta \rightarrow-\infty
$$

gives the third condition. The exponential decay of $\theta$ as $\eta \rightarrow-\infty$ means that the tail has both a negligible deflection from the vertical and negligible bending stress.

Equation (3.17) implies that $n_{3}=-\cos \theta$ at leading order, and so (3.24) implies $n_{3} \rightarrow-1$ as $\eta \rightarrow-\infty$, which is consistent with matching onto a vertical tail with a vertical compression $F_{z}$.

There is a unique solution to (3.21), (3.22) and (3.24). The scaled shape at the base of a slender dragged thread is therefore universal as $\epsilon \rightarrow 0$ when $U_{b}<U_{f}$. A variation of experimental parameters results in a simple rescaling of the size of the boundary layer according to the definition of $\delta_{z}$ in (3.19).

We note that the compressional-heel solution presented here also arises in the context of a dragged viscous sheet, which was analysed by Dyson (2007) in the regime where inertia is important; further discussion is given in Appendix A.

Figure 3(a) shows the shape of the universal solution, which we call the 'compressional' heel. We note that it has a backward-facing heel, which matches the observed behaviour of steadily dragged threads for slower belt speeds (cf. figure $1 b$ ). Above the backward-facing heel, there are exponentially damped oscillations about the vertical in agreement with (3.23), though these oscillations would be too small to be seen experimentally. The decay of the bending stresses away from the boundary 


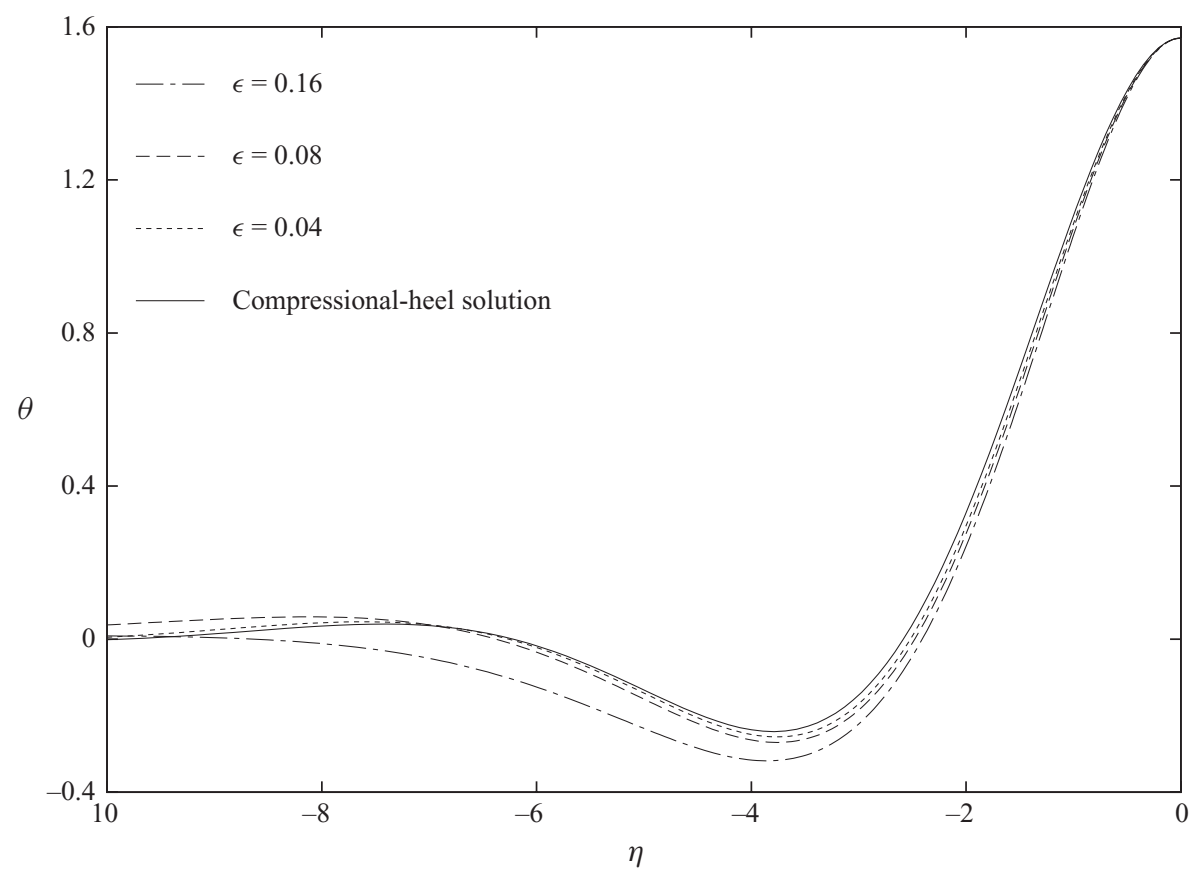

FiguRE 4. Convergence as $\epsilon$ decreases of numerical solution to the full system (2.13)-(2.20) of bending equations towards the asymptotic compressional-heel solution for the case $U_{n}=7.1 \times 10^{-3}$ and $U_{b}=0.25 U_{f}$. The values of $U_{n}$ and $\epsilon$ are representative of the experiments of CWL.

layer can be seen in figure $3(b)$. Figure 4 shows that the solutions to the full set of bending equations (2.13)-(2.20) converge towards the asymptotic solution given by (3.21), (3.22) and (3.24) as $\epsilon \rightarrow 0$. Values of $\epsilon$ corresponding to the experiments of CWL and Morris et al. (2008) range between $5 \times 10^{-4}$ and $4 \times 10^{-2}$, and there is very close agreement between the full and asymptotic solutions in this range. The full solutions are known to agree well with experiment (Ribe et al. 2006b), and hence the asymptotic solutions give good quantitative predictions for the observed heel shapes.

The gravitational term that was omitted from the force balance (3.16) to obtain (3.20) is $O\left(\pi a_{b}^{2} \delta_{z} / F_{z}\right)$ relative to the bending terms. Equation (3.19) implies that this omission is consistent provided

$$
\left(\frac{3 \pi a_{b}^{2} \epsilon^{2}}{4 F_{z}}\right)^{1 / 3} \ll \frac{F_{z}}{\pi a_{b}^{2}} .
$$

If $U_{b}<U_{f}$ is fixed then $a_{b}$ and $F_{z}$ are $O(1)$ as $\epsilon \rightarrow 0$ and (3.25) holds when $\epsilon$ is sufficiently small.

\subsection{Gravitational heels: $U_{b} \approx U_{f}$}

If $U_{b} \approx U_{f}$, then the thread undergoes only a small amount of compression relative to free-fall and $F_{z}$ is small. Hence, (3.25) breaks down when $U_{b} \rightarrow U_{f}$ with $\epsilon$ fixed, however small. This limit leads to a new regime in which gravity is also important near the belt.

We consider a distinguished double limit $\epsilon \rightarrow 0$ and $U_{b} \rightarrow U_{f}$ in which gravitational stresses are in balance with compressional and bending stresses throughout the boundary layer. The force balance (3.16) implies that the relevant force scale is 
$F_{g} \sim \pi a_{b}^{2} \delta_{g}$, where $\delta_{g}$ is the corresponding length scale. The small stresses within the boundary layer cause only a small deflection of the tail from vertical. Hence, the boundary layer must again deflect the thread from $\theta \approx 0$ in the tail to $\theta=\pi / 2$ at the belt, and the curvature $\kappa_{2}$ within the boundary layer again scales like $\theta^{\prime} \sim \delta_{g}^{-1}$. We substitute these scalings into the stress-moment balance (3.15) to obtain

$$
\delta_{g}=\left(\frac{3 \epsilon^{2}}{4}\right)^{1 / 4}, \quad F_{g}=\pi a_{b}^{2}\left(\frac{3 \epsilon^{2}}{4}\right)^{1 / 4} .
$$

(We note that these scalings coincide with those found by Ribe 2004 in the 'gravitational regime' of the related problem of steady fluid coiling on a stationary surface.)

Using $F_{g}$ and $\delta_{g}$ to define the rescaling (3.18), we rewrite (3.15) and (3.16) as

$$
\theta^{\prime \prime \prime}=-\phi_{z} \sin \theta-\phi_{x} \cos \theta-\eta \sin \theta,
$$

where $\phi_{x}$ and $\phi_{z}$ are constants given by

$$
\phi_{x}=\frac{F_{x}}{F_{g}} \quad \text { and } \quad \phi_{z}=\frac{F_{z}}{F_{g}} .
$$

Equation (3.27) links the values of $\phi_{x}$ and $\phi_{z}$ to the shape $\theta(\eta)$ of the thread near the belt. Hence, in contrast to the compressional heel, the values of $\phi_{x}$ and $\phi_{z}$ depend on the behaviour of the heel, and cannot be directly determined from consideration of the tail alone. As before, the two rolling boundary conditions

$$
\theta=\pi / 2 \text { and } \theta^{\prime}=0
$$

are imposed at the belt. Additional matching conditions are required to enforce the decay of $\theta$ and of bending stress into the tail. We again anticipate that $\theta \rightarrow 0$ as $\eta \rightarrow-\infty$, and linearise (3.27) about this value to obtain

$$
\theta^{\prime \prime \prime} \sim-\phi_{z} \theta-\phi_{x}-\eta \theta \text {. }
$$

The solution approaches

$$
\theta \sim-\frac{\phi_{x}}{\eta}+\sum_{i=1,2,3} A_{i} \exp \left(-\frac{3}{4} \lambda_{i} \eta^{4 / 3}\right) \quad \text { as } \quad \eta \rightarrow-\infty,
$$

where $\lambda_{i}^{3}=-1$, the first term on the right-hand side is a leading-order particular integral and the exponential modes are WKB approximations to the complementary function. Equation (3.31) implies that the growing exponential modes can be suppressed by imposing

$$
\theta \sim-\frac{\phi_{x}}{\eta} \quad \text { and } \quad \kappa_{2} \sim \frac{\phi_{x}}{\eta^{2}} \quad \text { as } \quad \eta \rightarrow-\infty .
$$

We remark that (3.32) is also satisfied by a tail that is governed by rescaled versions of (3.1)-(3.5) and deflected by a horizontal force $\phi_{x}$. Hence, (3.32) matches the horizontal force and deflection between the heel and the tail. The third-order equation (3.27) contains two free constants and is subject to the two boundary conditions (3.29) at the belt and the two matching conditions (3.32) that suppress the two divergent exponential modes in (3.31). Hence, there is a one-parameter family of solutions, which we call 'gravitational heels'. 
The value of the remaining parameter is determined by matching the vertical force between the heel and the tail. After rescaling, the vertical force balance $(3.14 b)$ is

$$
n_{1} \sin \theta-n_{3} \cos \theta=\phi_{z}+\eta \text {. }
$$

Since the deflection of the tail from vertical is small, the rescaled vertical coordinate $Z=z / \delta_{g}$ approaches $-\eta-\Delta \eta$ as $\eta \rightarrow-\infty$, where the offset $\Delta \eta$ is the extra arclength due to the curvature of the heel. Hence, as $\eta \rightarrow-\infty$ and $\theta \rightarrow 0$, the force balance (3.33) approaches

$$
n_{3}=Z-\Phi_{z}
$$

where the parameter

$$
\Phi_{z}=\phi_{z}-\Delta \eta
$$

is the effective vertical force exerted by the heel on the tail, given by the upward force from the belt less the extra weight in the heel. By matching to a vertical tail and substituting (3.3) and $F_{g}=\pi a_{b}^{2} \delta_{g}$ into (3.28b), we can determine $\Phi_{z}$ from the condition

$$
\Phi_{z}=\frac{3}{\delta_{g}} \frac{\mathrm{d} U}{\mathrm{~d} z} \quad \text { at } \quad z=0
$$

where $U(z)$ is the velocity profile in the nearly vertical tail.

This velocity profile must satisfy the vertical-fall equation (3.6), $U=U_{n}$ at the nozzle and $U=U_{b}$ at the belt. Since $U_{b} \approx U_{f}$, we estimate $\Phi_{z}$ by perturbing the free-fall velocity profile derived in $\S 3.1$. We anticipate that the belt exerts a small force on the tail and therefore substitute $T_{\infty}=\pi+t$, where $t \ll 1$, into (3.7) to obtain (omitting the $O(d)$ terms for simplicity)

$$
\left.\frac{\mathrm{d} U}{\mathrm{~d} z}\right|_{z=0}=\frac{1}{3 T_{\infty}} \sin T_{\infty}=-\frac{t}{3 \pi}+O\left(t^{2}\right) .
$$

A similar expansion for $U_{b}$ using (3.7) and (3.9) implies that

$$
U_{b}-U_{f}=-\frac{4 t}{3 \pi^{3}}+O\left(t^{2}\right)
$$

Combination of (3.36)-(3.38) together with $a_{b}^{2} \approx 1 / U_{f}$ yields

$$
\Phi_{z}=\frac{3 \pi^{2}}{4 \delta_{g}}\left(U_{f}-U_{b}\right)+O\left(\frac{\left(U_{f}-U_{b}\right)^{2}}{\delta_{g}}\right),
$$

thus relating the force $\Phi_{z}$ to the velocity difference $U_{f}-U_{b}$ that it must produce. We note that since $\delta_{g}=O\left(\epsilon^{1 / 2}\right)$, the estimate (3.39) is appropriate when $U_{b}-U_{f}=O\left(\epsilon^{1 / 2}\right)$.

Since $\Phi_{z}$ is determined by the tail, we use it to parametrise the family of heels, with the value of $\phi_{x}$ then being a consequence of the solution. Figure 5 shows some of the heel shapes for various values of $\Phi_{z}$, obtained by solving (3.27) numerically subject to (3.29), (3.32) and (3.34). Figure 6 shows the dependence of $\phi_{x}$ on $\Phi_{z}$. As $\Phi_{z}$ increases, the horizontal force $\phi_{x}$ tends to zero and the deflection of the tail from vertical thus also becomes small. For large values of $\Phi_{z}$, the thread shapes are similar to the shape of the compressional heel. This is because large values of $\Phi_{z}$ correspond to strong compression of the boundary layer, so that gravitational forces are negligible in comparison, $\Delta \eta \ll \phi_{z}$ and $\Phi_{z} \approx \phi_{z}$. A comparison of the length scales (3.19) and (3.26), together with (3.28b), shows that when $\Phi_{z} \gg 1$ the length scale of the gravitational heel is given by

$$
\delta_{g}=\Phi_{z}^{1 / 3} \delta_{z}
$$




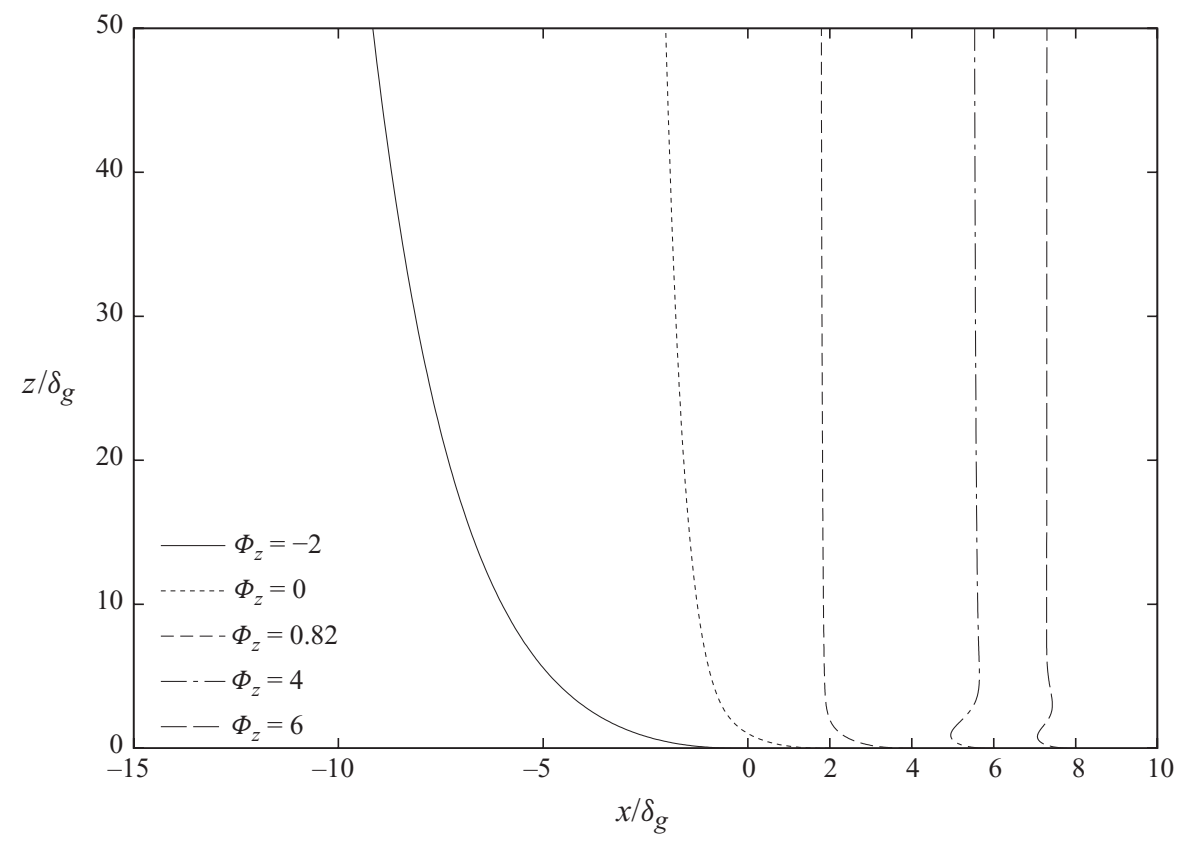

FIGURE 5. A plot of heels within the gravitational regime, for various values of the effective vertical force $\Phi_{z}$ on the tail. These shapes are obtained by solving (3.27) subject to the boundary conditions (3.29) and matching conditions (3.32) and (3.34) imposed at $\eta=-500$. The contact points are offset at multiples of 2 on the $x$-axis for clarity.

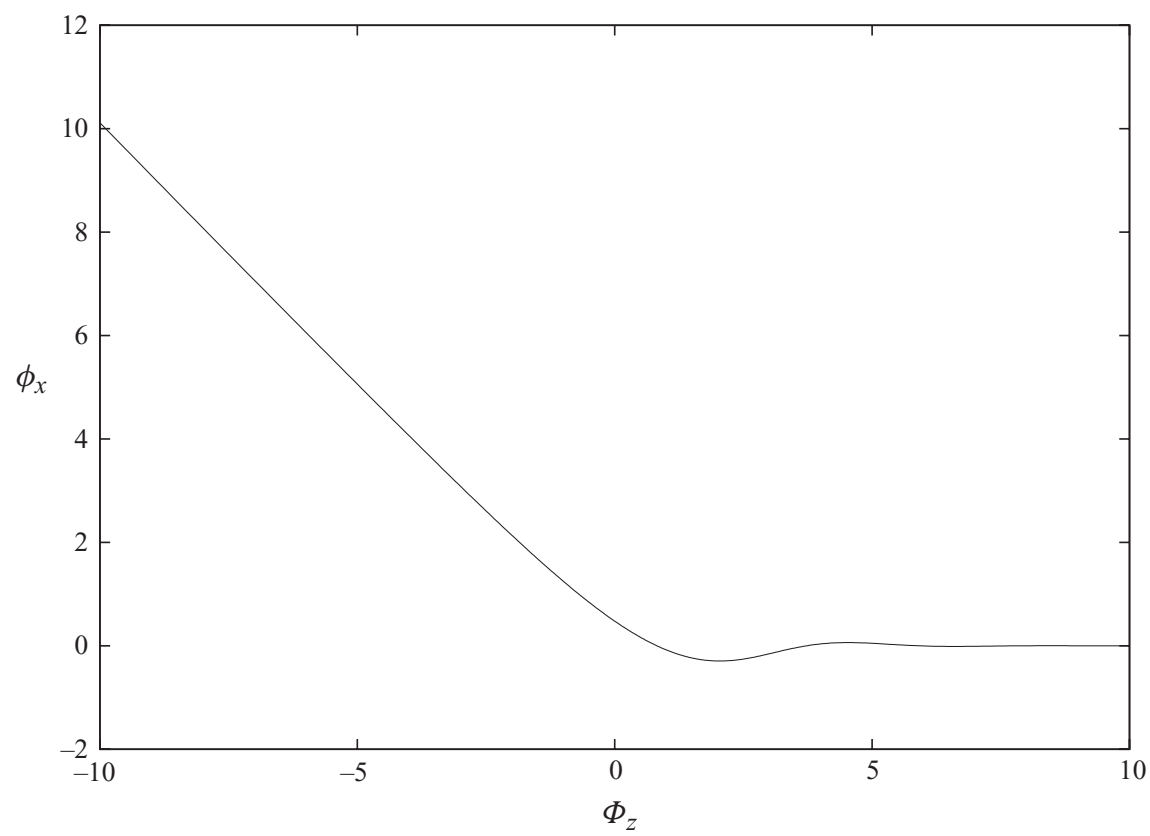

FIGURE 6 . The dependence of the rescaled horizontal force $\phi_{x}$ on $\Phi_{z}$. 


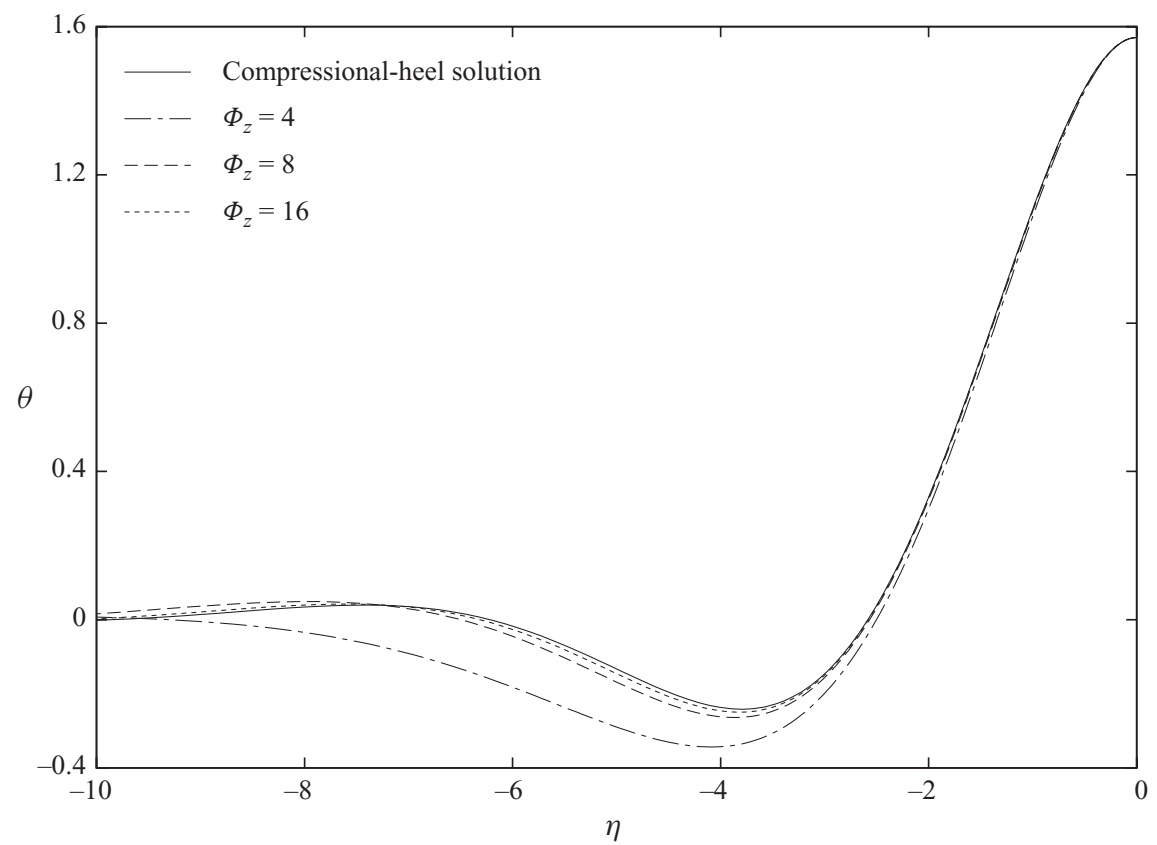

FIGURE 7. Convergence of rescaled solutions in the gravitational regime to the compressional-heel solution of $\S 3.3$ as $\Phi_{z} \rightarrow \infty$.

Figure 7 shows the convergence of the rescaled gravitational-heel solutions towards the compressional-heel solution in the limit $\Phi_{z} \rightarrow \infty$.

In the opposite limit, $\Phi_{z} \rightarrow-\infty$, figure 6 shows that bending stresses within the gravitational heel exert a large horizontal force $\phi_{x}$ on the tail. The deflection of the tail is therefore large and the shape of the thread (figure 5) increasingly resembles the catenary-like solutions found by CWL. This suggests that there is a third boundarylayer regime, which applies when $U_{b}>U_{f}$.

\section{5. $U_{b}>U_{f}$ : curvature-adjustment layer}

Previous approximate solutions that omit the effects of bending stress have been derived to describe threads for which $U_{b}>U_{f}$ (CWL; Hlod et al. 2007). While these solutions allow the thread to be horizontal at the bottom of the thread (unless inertia dominates), they also have a non-zero curvature $\kappa_{b}$ there. The role of bending stresses when $U_{b}>U_{f}$ is to adjust the curvature from $\kappa_{b}$ at the bottom of the tail to zero across the boundary layer, thus allowing all the dynamic rolling conditions to be satisfied at the belt. Outside this boundary layer, the bending stresses are unimportant and the solutions of CWL and Hlod et al. (2007) may be applied, after a small modification to the velocity condition which we will describe.

At the bottom of the tail where $\theta \approx \pi / 2$, the horizontal force balance $(3.14 a)$ implies that $N_{3} \approx F_{x}$. Hence, (3.4) implies that the curvature $\kappa_{b}$ at the bottom of the tail, above the boundary layer, is

$$
\kappa_{b}=\frac{\pi a_{b}^{2}}{F_{x}} .
$$

In contrast to the previous two regimes, the tail is nearly horizontal near the bottom, and $\theta$ does not vary significantly across the boundary layer. Hence, the curvature scaling is not given by an $O(1)$ variation in $\theta$ over an $O\left(\delta_{*}\right)$ length scale, but by $\kappa_{b}$. 
We aim to find the curvature $\kappa_{2}$ within the boundary layer. We combine (3.12) and (3.15) with $N_{3}=F_{x}$ and $\theta^{\prime}=\kappa_{2}$ to obtain

$$
\kappa_{2}-\frac{3 \pi a_{b}^{2} \epsilon^{2}}{4 F_{x}} \kappa_{2}^{\prime \prime \prime}=\kappa_{b} .
$$

Thus, the length scale of the boundary layer is given by

$$
\delta_{x} \sim\left(\frac{3 \pi a_{b}^{2} \epsilon^{2}}{4 F_{x}}\right)^{1 / 3}
$$

and, using this to define the rescaling $(3.18 a)$, we can rewrite $(3.42)$ as

$$
\kappa_{2}-\kappa_{2}^{\prime \prime \prime}=\kappa_{b}
$$

The general solution of (3.44) is

$$
\kappa_{2} \sim \kappa_{b}+\sum_{i=1,2,3} A_{i} \exp \left(-\lambda_{i} \eta\right),
$$

where $\lambda_{i}^{3}=-1$. The matching condition $\kappa_{2} \rightarrow \kappa_{b}$ as $\eta \rightarrow-\infty$ suppresses the two divergent modes in (3.45) and, together with the boundary condition $\kappa_{2}=0$ at $\eta=0$, defines a unique solution

$$
\kappa_{2}=\kappa_{b}\left(1-\mathrm{e}^{\eta}\right)
$$

which we call a 'curvature-adjustment layer'. This solution, like the compressional heel, is universal and qualitatively unchanged by variation of experimental parameters.

The variation of $\theta$ across the boundary layer is $O\left(\kappa_{b} \delta_{x}\right)$. From (3.41) and (3.43), the assumption that $\theta \approx \pi / 2$ throughout the boundary layer is consistent with this variation provided that

$$
\left(\frac{3 \pi a_{b}^{2} \epsilon^{2}}{4 F_{x}}\right)^{1 / 3} \ll \frac{F_{x}}{\pi a_{b}^{2}} .
$$

Hence, if $U_{b}>U_{f}$ is fixed, then $F_{x}$ and $a_{b}$ are fixed and (3.47) holds when $\epsilon$ is sufficiently small.

If $U_{b}$ is close to $U_{f}$, then there is only a small amount of stretching in the tail and $F_{x}$ is small. Hence, (3.47) breaks down when $U_{b} \rightarrow U_{f}$ with $\epsilon$ fixed, however small. The gravitational heels, which do not make the approximation $\theta \approx \pi / 2$ throughout the boundary layer near the belt, are applicable in this limit.

The gravitational-heel shapes for $\Phi_{z}<0$ in figure 5 suggest that the gravitational heels converge towards a curvature-adjustment layer as $\Phi_{z} \rightarrow-\infty$, and hence as $\phi_{x} \rightarrow \infty$. This convergence may be seen quantitatively by rescaling (3.46) with respect to the gravitational scales $F_{g}$ and $\delta_{g}$, to obtain

$$
\delta_{g} \kappa_{2}=\phi_{x}^{-1}\left(1-\mathrm{e}^{K \eta_{g}}\right)
$$

where

$$
\delta_{g}=\phi_{x}^{1 / 3} \delta_{x} \quad \text { and } \quad K=\phi_{x}^{1 / 3} .
$$

The convergence of gravitational heels towards this solution is shown in figure 8 .

We conclude that the gravitational heels match smoothly between the compressional heel, valid for fixed $U_{b}<U_{f}$ as $\epsilon \rightarrow 0$, and the curvature-adjustment solution, valid for fixed $U_{b}>U_{f}$ as $\epsilon \rightarrow 0$, with the width of the matching region given by $U_{b}$ $U_{f}=O\left(\epsilon^{1 / 2}\right)$. 


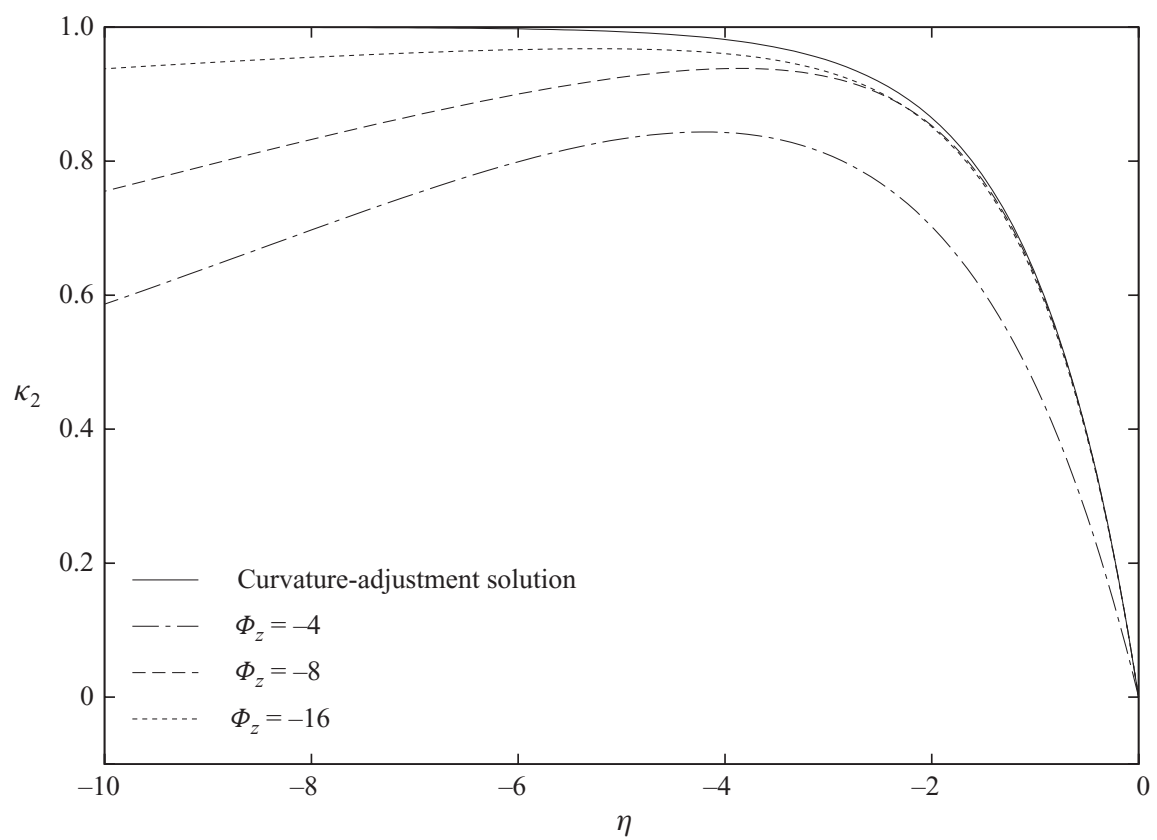

FIGURE 8 . Convergence of rescaled solutions in the gravitational regime to the curvature-adjustment solution (3.46) as $\Phi_{z} \rightarrow-\infty$.

\subsubsection{Boundary layer at the nozzle}

If bending effects are omitted, then the thread approaches the nozzle at some non-zero angle $\theta_{n}$ and the axial stress has a horizontal component $F_{x}$. The 'clamped' boundary conditions $(2.12 a, b)$ at the nozzle require there to be a bending boundary layer near the nozzle. Within this boundary layer, the force $F_{x}$ is supported by bending stress, and the orientation of the thread varies from $\theta=0$ at the nozzle towards $\theta=\theta_{n}$ in the tail. The analysis in $\S \S 3.3-3.5$ implies that $\theta_{n}$ remains $O(1)$ as $\epsilon \rightarrow 0$ only if $U_{b}>U_{f}$. Hence, the following analysis is relevant to the case $U_{b}>U_{f}$.

The horizontal force $F_{x}$ is determined, at leading order, by the stretching-dominated tail and is therefore $O(1)$ as $\epsilon \rightarrow 0$. In contrast, the gravitational stresses in the boundary layer at the nozzle are $O\left(\delta_{*}\right)$ and thus negligible as $\epsilon \rightarrow 0$. Hence, the analysis proceeds similarly to that of the compressional heel in $\S 3.3$, except with $\delta_{*}$ given by

$$
\delta_{n}=\left(\frac{3 \pi a_{n}^{2} \epsilon^{2}}{4 F_{n}}\right)^{1 / 3},
$$

where $F_{n}$ is the axial stress at the nozzle. The shape of the thread near the nozzle is governed (without rescaling) by

$$
\delta_{n}^{3} \theta^{\prime \prime \prime}-\sin \left(\theta-\theta_{n}\right)=0,
$$

with boundary conditions

$$
\theta=\theta^{\prime}=0 \quad \text { at } \quad s=-\ell \quad \text { and } \quad \theta \rightarrow \theta_{n} \quad \text { as } \quad \frac{s+\ell}{\delta_{n}} \rightarrow \infty .
$$

Linearisation of (3.51) about $\theta=\theta_{n}$ shows that the matching condition $\theta \rightarrow \theta_{n}$ as $(s+\ell) / \delta_{n} \rightarrow \infty$ suppresses one exponential mode and, together with (3.52), gives rise 
to a unique solution for each $\theta_{n}$. We note that there is one more boundary condition and one less matching condition than for the curvature-adjustment layer. This is due to the difference in direction between $(s+\ell) / \delta_{n} \rightarrow \infty$ and $s / \delta_{x} \rightarrow-\infty$ which requires different modes to be suppressed in order to match to the tail.

The length scale (3.50) implies that the bending-stress corrections at the nozzle are formally of the same order in $\epsilon$ as those at the belt. However, their numerical values are much smaller provided the belt speed is not so large that the horizontal stress $F_{x}$ exerted by the belt is comparable with the weight of the thread. It can be shown that in these circumstances the top of the tail hangs nearly vertically anyway and requires little change in curvature to match the boundary condition. Since the bending-stress corrections necessary to satisfy (3.52) at the nozzle are small, we neglect them in the calculations below.

\subsubsection{Perturbation to the tail}

Since $\theta=\pi / 2$ at the belt, $\kappa_{b}=\theta^{\prime}=O(1)$ and $\delta_{x} \ll 1$, the angle of the thread is given by $\theta \approx \pi / 2$ throughout the curvature-adjustment layer. Linearisation of (2.14) about this value yields $\theta \approx z^{\prime}$, and substitution of this result into (2.15) implies that the curvature is given by $\kappa_{2}=z^{\prime \prime}$ throughout the curvature-adjustment layer. The shape of the thread near the belt can therefore be found by integrating $z^{\prime \prime}=\kappa_{2}$ using (3.46) subject to $z=z^{\prime}=0$ at the contact point. We obtain

$$
z=\kappa_{b}\left(\frac{1}{2}(\eta+1)^{2}+\frac{1}{2}-\mathrm{e}^{\eta}\right),
$$

which approaches

$$
z \sim \frac{\kappa_{b}}{2}(\eta+1)^{2} \text { as } \eta \rightarrow-\infty .
$$

If the existence of the boundary layer is neglected, then extrapolation of (3.54) to $z=0$ gives contact at $\eta=-1$ rather than $\eta=0$; hence, the boundary layer provides an additional arclength of $\delta_{x}$. Since the thread continues stretching at a rate $U^{\prime}=F_{x} /\left(3 \pi a_{b}^{2}\right)$ between $\eta=-1$ and $\eta=0$, it follows that the effective boundary condition on the catenary solution in the tail is not $U=U_{b}$ at $z=0$, but instead $U=U_{b}-\Delta U$, where

$$
\Delta U=\frac{F_{x} \delta_{x}}{3 \pi a_{b}^{2}} .
$$

Together with (3.43), this implies that bending-stress corrections at the belt cause an $O\left(\epsilon^{2 / 3}\right)$ global perturbation to the tail, which dominates the $O\left(\epsilon^{2}\right)$ perturbations caused by the local bending stresses. This global perturbation makes a significant contribution to the dragout distance as described below.

\subsection{Dragout distance of asymptotic solutions and full numerical solutions}

We now use the asymptotic solutions found in the preceding sections to estimate the dimensionless dragout distance $x_{b}$, defined as the horizontal displacement from the nozzle to the contact point with the belt. We consider separately the contributions to $x_{b}$ that arise from the deflection of the tail from vertical and from the shape of the boundary layer at the belt. The boundary layer exerts a horizontal force $F_{x}$ on the tail that deflects it from vertical. Because of this force, the tail forms a catenary that hangs from the nozzle under gravity. We define $x_{t}$ to be the horizontal displacement between the nozzle and the minimum of this catenary extrapolated as if the boundary layer were not there. The curvature of the boundary layer modifies the shape of the thread near the belt from that of the tail. We define $x_{\ell}$ to be the distance between 
(a)

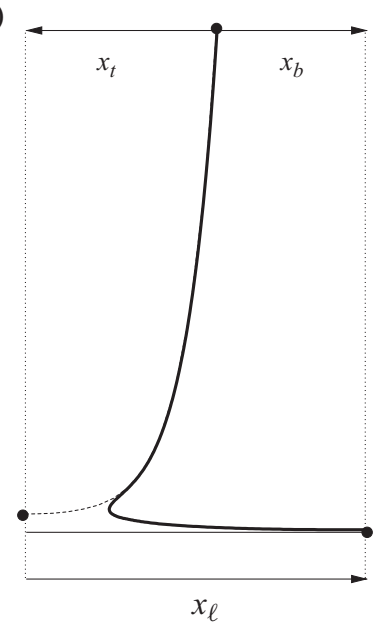

(b)

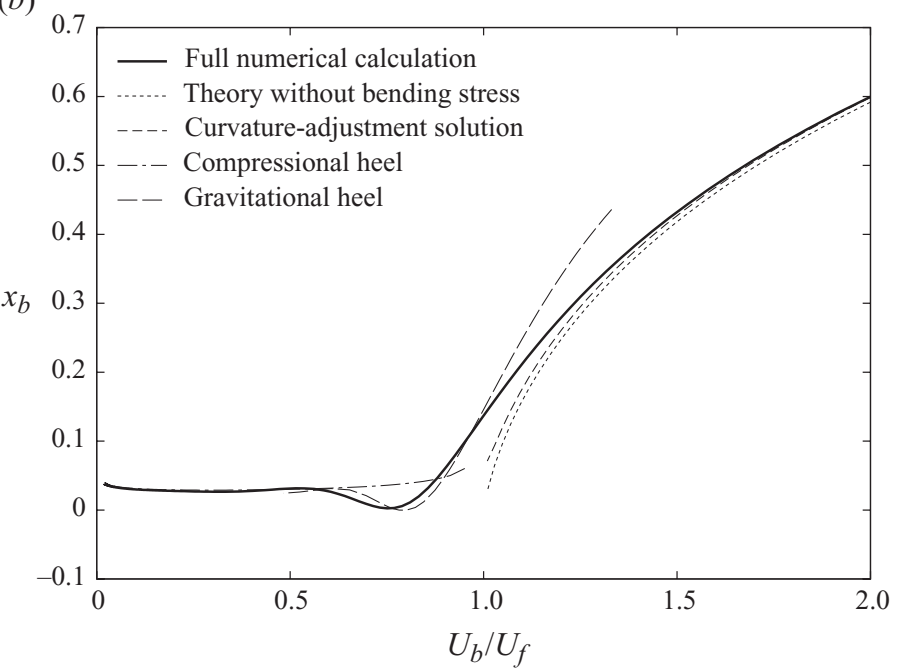

FiguRE 9. (a) The contributions to dragout distance from the heel and the stretchingdominated tail. The contribution $x_{t}$ from the tail is measured horizontally from the base of the extrapolated catenary (dashed) to the height of the nozzle, and $x_{\ell}$ is the offset from the catenary arising from the curvature of the heel (solid). (b) Asymptotic estimates of the dragout distance for various belt speeds compared with the values calculated using the full system of bending equations (2.13)-(2.20), for parameter values $\epsilon=4 \times 10^{-2}$ and $U_{n}=7 \times 10^{-3}$.

the minimum of the extrapolated catenary and the contact point with the belt. The contributions $x_{t}$ and $x_{\ell}$ to $x_{b}$ are illustrated in figure $9(a)$. Clearly, $x_{b}=x_{t}+x_{\ell}$.

The compressional heel matches to a tail that has negligible deflection from vertical and hence has $x_{t} \approx 0$. The contribution $x_{\ell}$ to the dragout distance of the compressional heel is found by numerical integration of (3.21) together with (2.1) to be $1.26 \delta_{z}$.

The gravitational heel matches to a tail with a deflection that is governed by the horizontal force $F_{x}=O\left(\epsilon^{1 / 2}\right)$ exerted by the boundary layer near the belt. We approximate the tail as a uniform catenary of weight $\pi a_{b}^{2}$ per unit length. This gives the leading-order estimate

$$
x_{t}=\frac{F_{x}}{\pi a_{b}^{2}} \ln \left(\frac{\ell}{F_{x}}\right)+O\left(F_{x}\right),
$$

where the arclength of the thread is $\ell=1+O\left(\epsilon^{1 / 2}\right)$. We show in Appendix B that the variation of thread radius towards the nozzle gives only an $O\left(F_{x}\right)$ contribution to $x_{t}$ and so has no effect at leading order. The additional offset $x_{\ell}$ is found by numerical integration of (3.27) and depends only on $\Phi_{z}$ and $\delta_{g}$.

The curvature-adjustment layer matches towards a tail for which the velocity condition at the bottom is modified to $U_{b}-\Delta U$ as described by (3.55), giving some displacement $x_{t}$. Since $\theta \approx \pi / 2$ throughout the curvature-adjustment layer, its leadingorder contribution $x_{\ell}$ is the additional arclength within the bending boundary layer, which was shown in $\S 3.5 .2$ to be $\delta_{x}$.

Figure $9(b)$ plots the various asymptotic estimates of $x_{b}$ and compares them to the full numerical solution. The parameter values used are $\epsilon=4 \times 10^{-2}$ and $U_{n}=7 \times 10^{-3}$, which are typical of the experiments performed by CWL and Morris et al. (2008). Surface tension and inertia have been suppressed here, but in Appendix A we describe how our results may be adapted to account for their effects. 
The estimates derived from the compressional heel and the curvature-adjustment layer give good agreement for small and large belt speeds, respectively. As anticipated, both estimates break down when $U_{b}$ is close to $U_{f}$. The estimates derived from the gravitational heels give good agreement for $U_{b} \approx U_{f}$.

Our estimates of $x_{b}$ improve those of CWL for $U_{b}>U_{f}$, by including the leadingorder $O\left(\epsilon^{2 / 3}\right)$ corrections arising both from the local modifications to the shape in the boundary layer at the belt and from the global modification to the shape of the tail caused by the increased length for stretching near the belt. Moreover, our theory provides solutions for $U_{b}<U_{f}$, where the theory of CWL could not, as the bending stresses within the gravitational and compressional heels are necessary in order to support the compression required to match towards the tail.

\section{Stability analysis of a dragged thread}

We now consider the onset of unsteady motion of the dragged thread. When the thread is steady, it lies completely in the $x-z$ plane. Hence, small perturbations to the steady motion decouple into two systems, which correspond respectively to 'outof-plane' perturbations in the $y$-direction and to 'in-plane' perturbations in the $x-z$ plane. The experiments of CWL showed that near onset, the thread meanders across the belt (figure $1 d$ ), which suggests that the primary instability corresponds to the out-of-plane system. Ribe et al. (2006b) used a slender-thread model to perform a linear stability analysis of the steady state, and obtained very good agreement with experiment for both the onset and the frequency of meandering at onset. Morris $e t$ al. (2008) demonstrated experimentally that the onset of meandering is well described as an out-of-plane Hopf bifurcation from the steady state.

In order to determine the key physical processes that govern the onset of meandering, we re-examine the model of Ribe et al. (2006b) in the asymptotic limit of a very slender thread. We find that during meandering, bending forces in the heel cause it to move sideways and away from beneath the nozzle. As the heel moves further away from the nozzle, the consequent deflection of the tail from vertical causes the nozzle to exert an increasing restoring force on the thread. When this force is sufficiently large, the heel starts to be pulled back towards $y=0$. As the heel returns, its deformation introduces bending and twisting, which provide the disturbance that causes the heel to buckle again during the next half-oscillation. The interaction between the bending and twisting forces in the heel and the restoring tension in the tail determines the frequency of meandering oscillations. By matching both force and displacement between the heel and the tail, we will obtain an asymptotic estimate for the meandering frequency and the linearised growth rate near onset, and hence deduce an asymptotic estimate for the boundary between stable steady states and meandering threads. The analysis builds on many of the ideas developed in $\S 3$. In particular, we find that the onset of meandering occurs within the gravitational-heel regime. This might be anticipated on the grounds that the onset of instability is likely to occur when some, but not much, of the thread is under compression. It follows that the compressional-heel solutions in $\S 3.3$ are unstable to meandering and that the dragged-catenary solutions in $\S 3.5$ are stable to meandering.

\subsection{Perturbation system}

In order to analyse the stability of a gravitational heel, we rescale the variables in the same way as in $\S 3.4$. The effect is that velocities are non-dimensionalised with the 
belt speed $U_{B}$, radii with the radius $a_{B}$ of the thread at the belt, axial lengths with

$$
\delta_{G}=\delta_{g} H=\left(\frac{3 a_{E}^{2} H^{2}}{4}\right)^{1 / 4},
$$

angular velocities and growth rate with $U_{B} / \delta_{G}$, stresses with $\mu \pi a_{B}^{2} U_{E} \delta_{G} / H^{2}$ and stress moments with $\mu \pi a_{B}^{2} U_{E} \delta_{G}^{2} / H^{2}$. The rescaled arclength of the heel is now $O(1)$, while the rescaled length of the tail is now

$$
L_{\delta}=\frac{L}{\delta_{G}}=\left(\frac{4}{3 \epsilon^{2}}\right)^{1 / 4} \frac{L}{H},
$$

which is $O\left(\epsilon^{-1 / 2}\right)$ as $\epsilon \rightarrow 0$.

We analyse the unsteady motion of the thread by seeking eigenmodes of the linearised equations for unsteady perturbations to a steadily dragged thread. The relevant perturbation variables, which concern only out-of-plane motion, are $y, d_{1 y}$, $d_{3 y}, \kappa_{1}, \kappa_{3}, M_{1}, M_{3}$ and $N_{2}$, where $d_{1 y}=\boldsymbol{d}_{1} \cdot \boldsymbol{e}_{y}$ and $\kappa_{1}=\boldsymbol{\kappa} \cdot \boldsymbol{d}_{1}$ etc. We denote the structure of the eigenmodes by, for example, $y=\hat{y}(s) \mathrm{e}^{\sigma t}$, where a hat denotes the complex amplitude and $\sigma$ is the complex growth rate of the perturbation. We also now denote the steady variables by overbars.

The onset of unsteady motion of a slender dragged thread has previously been analysed by Ribe et al. (2006b). We adapt their equations slightly to suit the asymptotic analysis here. We continue to omit the effects of surface tension, inertia and the $O\left(\epsilon^{2}\right)$ terms that represent unimportant regular perturbations caused by bending stress. We again use a Lagrangian basis for which $\boldsymbol{d}_{3}$ is tangential to the thread, and $\boldsymbol{d}_{1}$ and $\boldsymbol{d}_{2}$ lie within the cross-section of the thread. The shape of the thread and the orientation of the basis vectors are governed by

$$
\begin{aligned}
\hat{y}^{\prime} & =\hat{d}_{3 y}, \\
\hat{d}_{1 y}^{\prime} & =-\bar{\kappa}_{2} \hat{d}_{3 y}+\hat{\kappa}_{3} \bar{d}_{2 y}, \\
\hat{d}_{3 y}^{\prime} & =\bar{\kappa}_{2} \hat{d}_{1 y}-\hat{\kappa}_{1} \bar{d}_{2 y},
\end{aligned}
$$

where $d_{i j}=\boldsymbol{d}_{i} \cdot \boldsymbol{e}_{j}$ and the equations correspond to the linearisation of $\boldsymbol{x}^{\prime}=\boldsymbol{d}_{3}$ and $\boldsymbol{d}_{i}^{\prime}=\boldsymbol{\kappa} \times \boldsymbol{d}_{i}$.

The dynamic behaviour of the thread is governed by

$$
\begin{aligned}
\hat{\omega}_{1}^{\prime} & =\frac{\hat{M}_{1}}{\bar{a}^{4}}+\sigma \bar{\kappa}_{2} \hat{d}_{1 y}, \\
\hat{\omega}_{3}^{\prime} & =\frac{3 \hat{M}_{3}}{2 \bar{a}^{4}}+\sigma \bar{\kappa}_{2} \hat{d}_{3 y}, \\
\hat{N}_{2}^{\prime} & =\bar{N}_{3} \hat{\kappa}_{1}-\bar{N}_{1} \hat{\kappa}_{3}+\bar{a}^{2} \hat{d}_{2 z}, \\
\hat{M}_{1}^{\prime} & =\bar{M}_{2} \hat{\kappa}_{3}-\bar{\kappa}_{2} \hat{M}_{3}+\hat{N}_{2}, \\
\hat{M}_{3}^{\prime} & =\bar{\kappa}_{2} \hat{M}_{1}-\bar{M}_{2} \hat{\kappa}_{1},
\end{aligned}
$$

where $\hat{\omega}_{1}=\bar{U} \hat{\kappa}_{1}+\sigma \hat{d}_{3 y}$ and $\hat{\omega}_{3}=\bar{U} \hat{\kappa}_{3}-\sigma \hat{d}_{1 y}$ are angular velocities that may be obtained by linearisation of $\mathrm{D} \boldsymbol{d}_{i} / \mathrm{D} t=\boldsymbol{\omega} \times \boldsymbol{d}_{i}$. Equations (4.6) and (4.7) are constitutive relations, and we have again omitted small contributions to the stress moment from coupling between stretching and bending (Entov \& Yarin 1984; Yarin 1993) on the grounds that curvature variations occur on the boundary-layer scale and velocity variations over the much longer fall height. Equation (4.8) is a stress balance, and (4.9) and (4.10) are stress-moment balances. 
As in the steady problem, the angular velocity and velocity of the thread are continuous at the contact point with the belt, and hence

$$
\hat{\omega}_{1}=\hat{\omega}_{3}=0 \quad \text { and } \quad \frac{\bar{U}}{\bar{U}_{B}} \boldsymbol{d}_{3}+\partial y / \partial t \boldsymbol{e}_{y}=\boldsymbol{e}_{x} \quad \text { at } \quad s=0 .
$$

We now fix the orientation of $\boldsymbol{d}_{1}$ and $\boldsymbol{d}_{2}$ within the cross-section by imposing $\boldsymbol{d}_{1}=\boldsymbol{e}_{z}$ at the contact point. By eliminating $\hat{\omega}_{i}$ in favour of $\hat{\kappa}_{i}$, substituting $\bar{U}=\bar{U}_{B}$ and projecting $(4.11 c)$ onto $\boldsymbol{e}_{y}$, we obtain the boundary conditions

$$
\hat{\kappa}_{1}=-\sigma \hat{d}_{3 y}, \quad \hat{\kappa}_{3}=0, \quad \sigma \hat{y}=-\hat{d}_{3 y} \quad \text { and } \quad \hat{d}_{1 y}=0 \quad \text { at } \quad s=0 . \quad(4.12 a-d)
$$

Similarly, the boundary conditions at the nozzle are that the position, orientation and angular velocity of the thread are continuous. Hence,

$$
\hat{y}=0, \quad \hat{d}_{3 y}=\hat{\omega}_{1}=0 \quad \text { and } \quad \hat{\omega}_{3}=0 \quad \text { at } \quad s=-L_{\delta} .
$$

Equations (4.3)-(4.10) subject to the eight boundary conditions (4.12) and (4.13) are an eigenvalue problem, which is linear in the perturbation variables and has non-trivial solutions only for discrete values of the growth rate $\sigma$. This full problem is solved numerically using the procedure described by Ribe et al. (2006b). The eigenmode is determined up to a multiplicative constant.

\subsection{Asymptotic solution for the perturbation eigenmode as $\epsilon \rightarrow 0$}

By analogy with a steadily dragged thread, we anticipate that the bending and twisting stresses in a meandering thread are negligible as $\epsilon \rightarrow 0$, except within a small boundary layer near the belt. Thus, the eigenmode also divides asymptotically into heel and tail regions, which are related by matching. The matching conditions (3.32) imply that the steady-state variables $\bar{\kappa}_{2}, \bar{M}_{2}$ and $\bar{N}_{1}$ all decay away from the heel. It follows that (4.6), (4.8) and (4.9) reduce to equations for the out-of-plane bending variables $\hat{\omega}_{1}, \hat{M}_{1}$ and $\hat{N}_{2}$ that are equivalent to the in-plane bending equations that gave (3.27). There are therefore two out-of-plane bending modes, analogous to those in (3.31), that do not decay away from the heel and must be suppressed by imposing

$$
\hat{d}_{3 y}=-\frac{\hat{\phi}_{y}}{\eta}+o\left(\frac{1}{\eta}\right) \quad \text { and } \quad \hat{d}_{3 y}^{\prime}=\frac{\hat{\phi}_{y}}{\eta^{2}}+o\left(\frac{1}{\eta^{2}}\right), \quad 1 \ll-\eta \ll L_{\delta} .
$$

These matching conditions are analogous to the steady in-plane conditions (3.32). We also need a condition on the twisting mode. We will see that meanders with amplitude $\hat{A}$ produce an $O\left(\hat{A} \sigma^{2}\right)$ twisting rate $\hat{\omega}_{3}$. In order to satisfy $\hat{\omega}_{3}=0$ at the nozzle, this twisting rate must be modified across the $O\left(L_{\delta}\right)$ length of the tail, and hence it follows that

$$
\hat{M}_{3}=O\left(\hat{A} \sigma^{2} / L_{\delta}\right), \quad 1 \ll-\eta \ll L_{\delta}
$$

An additional condition is obtained by matching the out-of-plane components of stress and displacement. As the heel meanders, the bending stresses in the heel exert a force $\hat{\phi}_{y}=\bar{N}_{1} \hat{d}_{1 y}+\hat{N}_{2} \bar{d}_{2 y}+\bar{N}_{3} \hat{d}_{3 y}$ on the base of the tail and the motion of the heel requires a deflection $\hat{y}_{t}$ of the base of the tail. The relationship between this out-of-plane force and deflection is analogous to the relationship (3.56) between the in-plane variables $\bar{\phi}_{x}$ and $\bar{x}_{t}$ in the steady state. Hence,

$$
\hat{y}_{t}=\hat{\phi}_{y} \ln L_{\delta}+O\left(\hat{\phi}_{y} \ln \hat{\phi}_{y}\right)
$$


The correction term is asymptotically negligible. Since $\hat{y}=0$ where the thread is pinned at the nozzle, (4.15) implies the constraint

$$
\hat{y}_{b}=\hat{\phi}_{y} \ln L_{\delta}+\hat{y}_{\ell}
$$

where $\hat{y}_{b}$ represents the out-of-plane displacement of the contact point with the belt, and $\hat{y}_{\ell}$ represents the contribution from the out-of-plane curvature in the heel.

The matching conditions (4.14) and (4.16) are applied towards the tail and replace the boundary conditions (4.13) that were imposed at the nozzle. The eigenmode is again determined up to a multiplicative constant, which we now set by imposing a fixed amplitude of oscillations, given by $\hat{y}_{b}=\hat{A}$. This amplitude is independent of $\sigma$, and hence the only contribution to the out-of-plane displacement at the belt appears at leading order, with no contributions at $O(\hat{A} \sigma)$ or higher.

We consider the out-of-plane motion of the heel near onset, so that $\operatorname{Re}(\sigma) \ll 1$. Then (4.16) implies that $\hat{\phi}_{y}=O\left(\hat{A} / \ln L_{\delta}\right)$. Hence, as $\epsilon \rightarrow 0$ so that $L_{\delta} \rightarrow \infty$, a fixed meandering amplitude requires an asymptotically small restoring force. We therefore expect $\operatorname{Im}(\sigma) \ll 1$ for a very slender thread. This motivates expansion of the eigenmode in powers of $\sigma$ for $|\sigma| \ll 1$. Since formally setting $\sigma=0$ represents a steady solution, the leading-order behaviour of the eigenmode at $O\left(\hat{A} \sigma^{0}\right)$ is steady. The steady solution that satisfies (4.3)-(4.10) and the conditions (4.16), (4.12) and (4.14) is simply a uniform displacement of the entire heel with $\hat{y}=\hat{A}$ and with all other perturbation variables vanishing. This displacement is due to deflection of the tail, and thus there is no contribution to $\hat{y}_{\ell}$ at this order. Hence, $\hat{y}_{\ell}=O(\hat{A} \sigma)$.

The $O(\hat{A} \sigma)$ contribution to the eigenmode is forced only through the condition (4.12c) that $\sigma \hat{y}=-\bar{U} \hat{d}_{3 y}$ at the belt, which corresponds to a quasi-steady translation of the contact point with velocity $\hat{A} \sigma$. This forced problem is equivalent to a small change to the direction of belt motion, for which the solution is simply a rotation of the steady heel about the vertical axis through an angle $\hat{A} \sigma$. We therefore pose the solution

$$
\begin{gathered}
\hat{y}=\hat{A}-\hat{A} \sigma\left(\bar{x}-\bar{x}_{b}\right)+O\left(\hat{A} \sigma^{2}\right), \\
\hat{d}_{3 y}=-\hat{A} \sigma \bar{d}_{3 x}+O\left(\hat{A} \sigma^{2}\right), \\
\hat{d}_{1 y}=-\hat{A} \sigma \bar{d}_{1 x}+O\left(\hat{A} \sigma^{2}\right) \\
\hat{\kappa}_{1}, \hat{M}_{1}, \hat{N}_{2}, \hat{\kappa}_{3}, \hat{M}_{3} \sim O\left(\hat{A} \sigma^{2}\right) .
\end{gathered}
$$

It is easy to verify that this solution satisfies the perturbation equations (4.3)-(4.10) and all boundary and matching conditions to $O(\hat{A} \sigma)$. The uniform translation $\hat{A} \sigma \bar{x}_{b}$ is included in (4.17) so that $\hat{y}_{b}$ remains equal to $\hat{A}$.

Since $\hat{A}$ is defined by $\hat{y}_{b}=\hat{A}$, the only contribution to $\hat{y}_{b}$ is at $O\left(\hat{A} \sigma^{0}\right)$ and there is no contribution at $O(\hat{A} \sigma)$ or higher. The $O\left(\hat{A} \sigma^{2}\right)$ terms in the eigenmode are forced only through the condition $(4.12 a)$ that $\bar{U} \hat{\kappa}_{1}=-\sigma \hat{d}_{3 y}$ at the belt. The $O\left(\hat{A} \sigma^{2}\right)$ contribution is not a simple geometrical operation and must be determined numerically. The $O\left(\hat{A} \sigma^{3}\right)$ terms in the eigenmode are forced both at the belt through $(4.12 a)$ and internally through (4.6) and (4.7). Neither the $O(\hat{A})$ displacement nor the $O(\hat{A} \sigma)$ rotation involve twisting, justifying the estimate $(4.14 c)$ of the twisting stress in the tail.

\subsection{Estimation of onset and frequency of instability}

Equation (4.17) and the definitions of $\hat{y}_{t}$ and $\bar{x}_{t}$ give

$$
\hat{y}_{t}=\hat{A}-\hat{A} \sigma \bar{x}_{t}+O\left(\hat{A} \sigma^{2}\right) .
$$


Equations (4.18)-(4.20) and the definition of $\hat{\phi}_{y}$ imply that

$$
\hat{\phi}_{y}=-\hat{A} \sigma \bar{\phi}_{x}-\hat{A} \sigma^{2} G_{0}+O\left(\hat{A} \sigma^{3}\right),
$$

where the $O(1)$ constant $G_{0}$ depends only on the shape of the steady heel and can be determined numerically from the $O\left(\hat{A} \sigma^{2}\right)$ contribution to the eigenmode. If we select the phase of the oscillation so that $\hat{A}$ is real, then the expansion of the eigenmode in powers of $\sigma$ yields systems of equations that have real coefficients. Hence, the solution at each power of $\sigma$ is real, and $G_{0}$ is also real.

Substitution of (4.21) and (4.22) into (4.16) yields

$$
\hat{A}=-\hat{A} \sigma \bar{\phi}_{x} \ln L_{\delta}-\hat{A} \sigma^{2} G_{0} \ln L_{\delta}+O\left(\hat{A} \sigma, \hat{A} \sigma^{3} \ln L_{\delta}\right) .
$$

If $\bar{\phi}_{x} \neq 0$, then a leading-order balance would imply that $\sigma=-1 /\left(\bar{\phi}_{x} \ln L_{\delta}\right)$. If $\bar{\phi}_{x}$ is held constant as $L_{\delta} \rightarrow \infty$, then solution at successive powers of $\sigma$ would further imply that the meandering frequency $\operatorname{Im}(\sigma)$ is $O\left(1 / \ln L_{\delta}^{3}\right)$. Hence, the eigenmode would grow or decay far faster than it would oscillate as $L_{\delta} \rightarrow \infty$, which is not what is observed experimentally near onset.

Since we wish to determine the behaviour near onset, we require $\operatorname{Re}(\sigma) \ll \operatorname{Im}(\sigma) \ll 1$. To this end, we consider a distinguished limit with $\bar{\phi}_{x}=F_{0} / \sqrt{\ln L_{\delta}}+O\left(1 / \ln L_{\delta}\right)$ as $L_{\delta} \rightarrow \infty$. Substitution into (4.23) yields

$$
1=-\sigma F_{0} \sqrt{\ln L_{\delta}}-\sigma^{2} G_{0} \ln L_{\delta}+O\left(\sigma, \sigma^{3} \ln L_{\delta}\right),
$$

and hence

$$
\sigma=\frac{-F_{0} \pm \mathrm{i} \sqrt{4 G_{0}-F_{0}^{2}}}{2 G_{0} \sqrt{\ln L_{\delta}}}+O\left(\frac{1}{\ln L_{\delta}}\right) .
$$

We see from (4.25) that the onset of meandering $(\operatorname{Re}(\sigma)=0)$ corresponds to $F_{0}=0$, and hence the marginally stable steady shape has $\bar{\phi}_{x}=\bar{\phi}_{x}^{*}$, where $\bar{\phi}_{x}^{*}=O\left(1 / \ln L_{\delta}\right)$ as $L_{\delta} \rightarrow \infty$. The meandering frequency at onset is given by

$$
\sigma^{*}= \pm \frac{\mathrm{i}}{\sqrt{G_{0} \ln L_{\delta}}}+O\left(\frac{1}{\ln L_{\delta}}\right)
$$

\subsection{Quantitative estimates}

The value of $G_{0}$ depends on the steady shape of the heel, and therefore on $\bar{\phi}_{x}$. For the moment, we make the approximation that $\bar{\phi}_{x}^{*}=0$ rather than $O\left(1 / \ln L_{\delta}\right)$. Under this approximation, (4.23) remains accurate to $O\left(1 / \sqrt{\ln L_{\delta}}\right)$.

Figure 6 shows that there are many heel solutions for which $\bar{\phi}_{x}=0$. However, we expect that the initial onset of meandering, as $\bar{U}_{b}$ is reduced, corresponds to the first of these heels which is under the smallest amount of vertical compression. This heel has $\bar{\Phi}_{z}=0.82$, and is shown in figure 5. If the belt speed is reduced slightly further, so that $\bar{\Phi}_{z}$ has a slightly larger value, then figure 6 shows that $\bar{\phi}_{x}<0$. Hence, $F_{0}<0$, and (4.25) implies that the heel is unstable to meandering at the reduced speed, which is in agreement with the experimentally observed direction of instability.

Substitution of the leading-order estimate $\bar{\Phi}_{z}^{*}=0.82$ into (3.39) implies that the onset of meandering occurs at a critical belt speed (in dimensional form)

$$
\bar{U}_{B}^{*}=\bar{U}_{F}\left(1-0.82 \frac{4 \delta_{g}}{3 \pi^{2}}+O\left(\frac{\epsilon^{1 / 2}}{\ln \epsilon}\right)\right),
$$




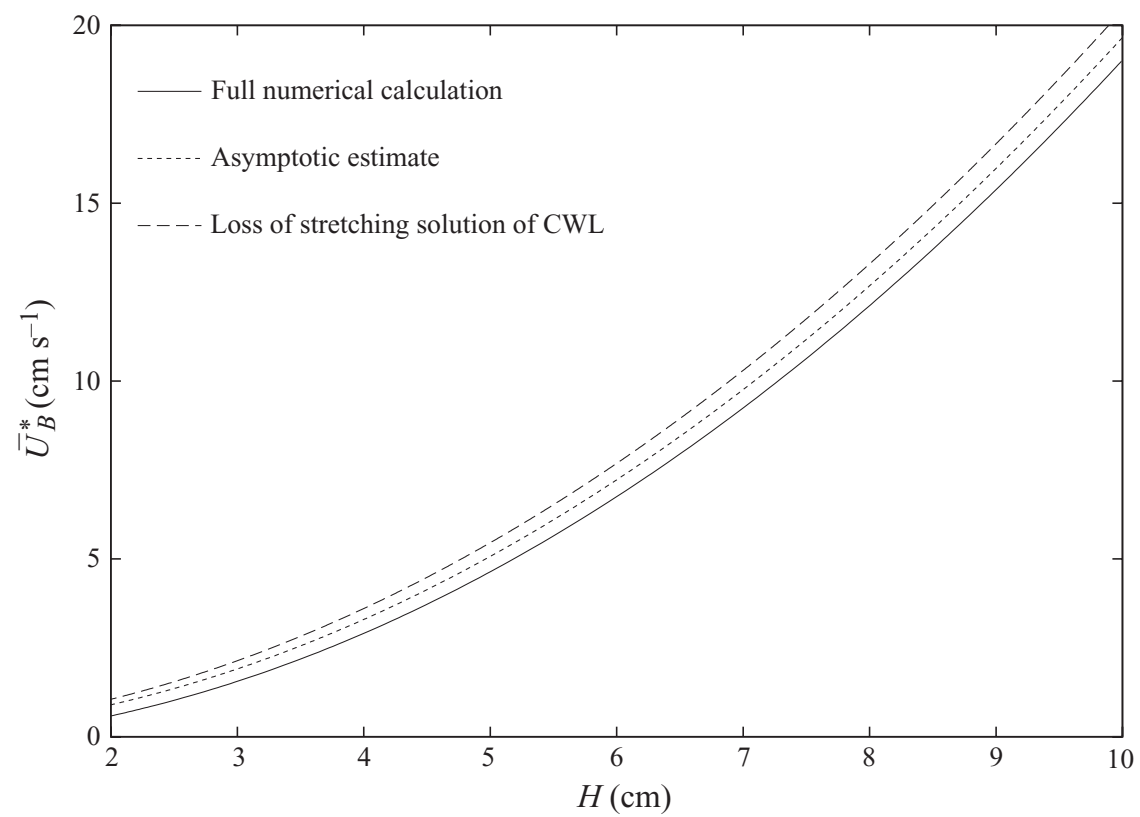

FigurE 10. Asymptotic estimate (4.27) of the neutrally stable belt speed $\bar{U}_{B}^{*}$ compared with the full numerical solution of (4.3)-(4.10), (4.12) and (4.13), for parameter values $Q=0.044 \mathrm{~cm}^{3} \mathrm{~s}^{-1}$, $a_{N}=0.5 \mathrm{~cm}$ and $v=347 \mathrm{~cm}^{2} \mathrm{~s}^{-1}$ which correspond to experiment 5 of CWL. (Surface tension and inertia are omitted in both the estimate and the numerical solution.) There is reasonable agreement, despite the omission of higher-order corrections that are only $O\left(1 / \sqrt{\ln \delta_{g}}\right)$ smaller.

where

$$
\delta_{g}=\left(\frac{3 \epsilon^{2}}{4}\right)^{1 / 4}=\left(\frac{3 Q \mu}{4 \pi \rho g H^{4}}\right)^{1 / 4} .
$$

The value of $G_{0}=0.198$ may be obtained through numerical calculation for the heel with $\bar{\Phi}_{z}=0.82$. Substitution into (4.26) gives the dimensional meandering frequency at onset as

$$
\operatorname{Im}\left(\sigma^{*}\right)= \pm \frac{\bar{U}_{F}}{H \delta_{g}}\left(\frac{2.25}{\sqrt{\ln \delta_{g}}}+O\left(\frac{1}{\ln \delta_{g}}\right)\right)
$$

Figures 10 and 11 compare the values of $\bar{U}_{B}^{*}$ and $\operatorname{Im}\left(\sigma^{*}\right)$ calculated from the full numerical solution to the asymptotic estimates (4.27) and (4.29), for the parameter values corresponding to experiment 5 of CWL. The estimate of $\bar{U}_{B}^{*}$ improves on the corresponding estimate of CWL. The agreement is particularly good for large values of $H$ since $\epsilon=a_{E} / H \propto H^{-2}$. The estimate (4.29) predicts the qualitative dependence of $\sigma^{*}$ on $H$. The accuracy of the asymptotic estimates is reasonable given that the corrections to (4.27) and (4.29) are logarithmic in $\delta_{g}$ and hence decay slowly as $\epsilon \rightarrow 0$.

Equation (4.25) implies that the onset of meandering occurs when $\bar{\phi}_{x}^{*}=O\left(1 / \ln L_{\delta}\right)$. In order to derive a quantitative estimate for $\bar{\phi}_{x}^{*}$ when $L_{\delta} \gg 1$, we pose the expansion

$$
\bar{\phi}_{x}^{*}=\frac{F_{1}}{\ln L_{\delta}}+O\left(\frac{1}{\left(\ln L_{\delta}\right)^{3 / 2}}\right),
$$




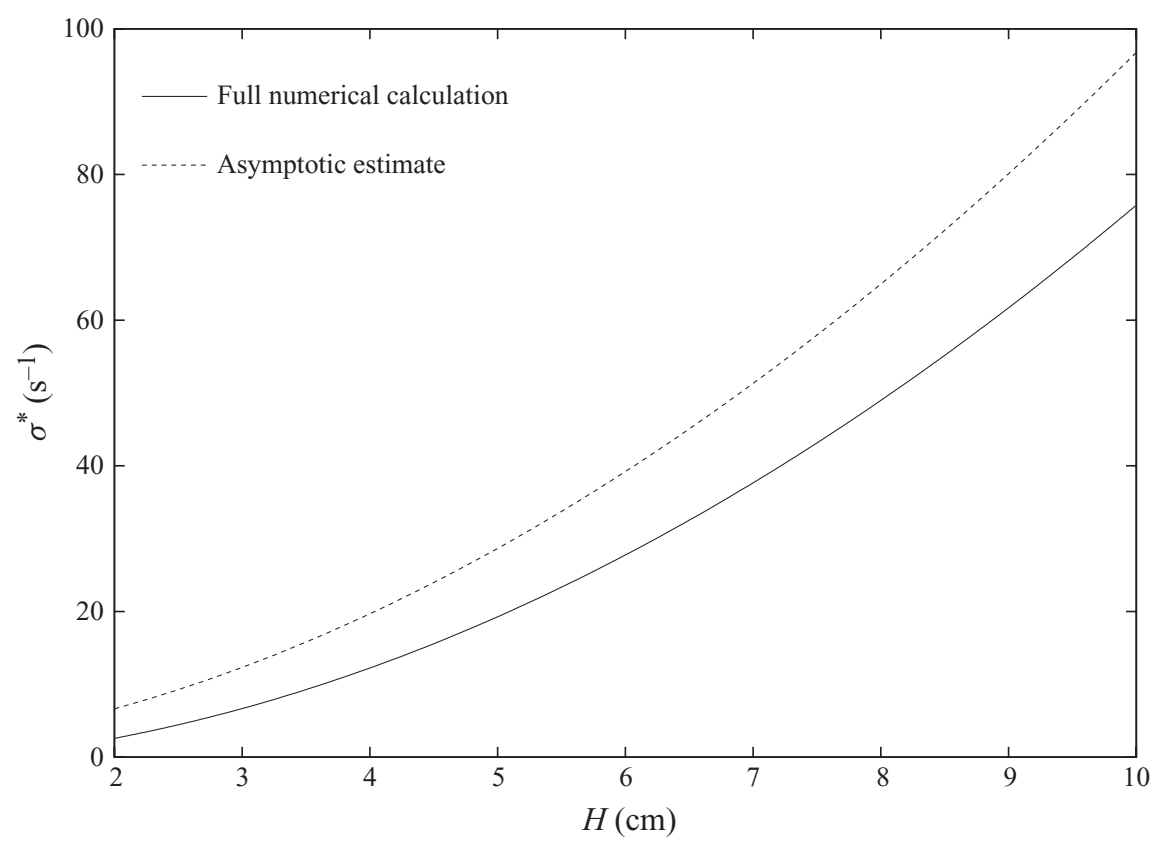

FIGURE 11. Leading-order asymptotic estimate (4.29) of the meandering frequency $\sigma^{*}$ at onset, compared with the full numerical solution of (4.3)-(4.10), (4.12) and (4.13), for parameter values $Q=0.044 \mathrm{~cm}^{3} \mathrm{~s}^{-1}, a_{N}=0.5 \mathrm{~cm}$ and $\nu=347 \mathrm{~cm}^{2} \mathrm{~s}^{-1}$ corresponding to experiment 5 of CWL. There is reasonable agreement, despite the omission of higher-order corrections that are only $O\left(1 / \sqrt{\ln \delta_{g}}\right)$ smaller.

for the steady state at onset, and extend the expansions

$$
\begin{aligned}
\hat{y}_{\ell} & =-\hat{A} \sigma \bar{x}_{\ell}+O\left(\hat{A} \sigma^{2}\right), \\
\hat{\phi}_{y} & =-\hat{A} \sigma \bar{\phi}_{x}+\hat{A} G_{0} \sigma^{2}+\hat{A} G_{1} \sigma^{3}+O\left(\hat{A} \sigma^{4}\right), \\
\sigma & =\frac{\mathrm{i}}{G_{0} \sqrt{\ln L_{\delta}}}+\frac{\sigma_{1}}{\ln L_{\delta}}+O\left(\frac{1}{\left(\ln L_{\delta}\right)^{3 / 2}}\right),
\end{aligned}
$$

for the relevant parts of the eigenmode and $\sigma$. Substitution of (4.30) into (4.16) yields

$$
F_{1}+\bar{x}_{\ell}+2 \sigma_{1} G_{0}-\frac{G_{1}}{G_{0}}=0 .
$$

The parameters $G_{0}$ and $G_{1}$ again depend on $\bar{\phi}_{x}$, and hence on $F_{1}$. However, calculation of $G_{0}, G_{1}$ and $\bar{x}_{\ell}$ with $F_{1}=0$ introduces only an $O\left(\hat{A} \sigma^{3}\right)$ error in $(4.30 b)$ and an $O\left(\hat{A} \sigma^{4}\right)$ error in $(4.30 c)$, which does not affect the calculation of $\sigma_{1}$. We therefore make this approximation and calculate $\bar{x}_{\ell}=2.08$ and $G_{1}=0.220$ for the heel with $\bar{\Phi}_{z}=0.82$. The leading-order estimate for $\bar{\phi}_{x}^{*}$ at onset of meandering is then obtained by setting $\sigma_{1}=0$ to give

$$
\bar{\phi}_{x}^{*}=-\frac{0.969}{\ln L_{\delta}}+O\left(\ln L_{\delta}^{-3 / 2}\right) .
$$

Since $\bar{\phi}_{x}^{*}$ is negative, it corresponds to a steady shape with a backward-facing heel, in agreement with experimental observations near the onset of meandering. 


\section{Discussion}

In this paper, we have demonstrated the importance of the bending resistance of a steadily dragged viscous thread to its motion. Our asymptotic analysis for a very slender thread has determined the leading-order dynamic effects of bending stress. We have shown that there are three distinct regimes for the shape of the thread, corresponding to the belt speed $U_{B}$ being less than, greater than or close to the 'free-fall' speed $U_{F}$, which we define as the fall speed under viscous-gravity balance of a thread that is stress-free at the fall height $H$ below the nozzle. If $U_{B}$ is larger than $U_{F}$, the analysis provides simple $O\left(\epsilon^{2 / 3}\right)$ corrections to calculations of catenary shapes that omit bending stress. If $U_{B}$ is smaller than $U_{F}$ then the analysis predicts the existence of the backward-facing heels observed at low belt speeds, which give an $O\left(\epsilon^{2 / 3}\right)$ correction to a vertically falling thread solution. We have also demonstrated the existence of a transitional regime, which applies when $U_{B}-U_{F}=O\left(\epsilon^{1 / 2}\right)$, in which the bending stresses give an $O\left(\epsilon^{1 / 2}\right)$ correction to the vertical thread solution. The smooth transition between this transitional regime and the other two regimes shows that the solutions cover the full range of steady shapes for a viscous thread. In all regimes, our analysis has isolated the key physical processes that govern bending at the bottom of the thread.

Our analysis of unsteady motion has provided a better understanding of the meandering instability observed experimentally, and yields simple asymptotic estimates (4.27) of the onset and (4.29) of the frequency of meandering. The onset of meandering occurs in the gravitational-heel regime of $\$ 3.4$ so that the compressionalheel solutions in $\S 3.3$ are unstable and that the dragged-catenary solutions in $\S 3.5$ are stable. The pinning of the thread at the nozzle, the physical effects of which had not previously been considered, plays a crucial role, since the scaling of the meandering frequency is determined from the restoring force generated through deflection of the tail and thus contains a logarithmic factor from this catenary-like deflection.

At leading order, our asymptotic model predicts that neutral stability occurs when the horizontal force $\bar{\phi}_{x} \approx 0$ and the vertical force $\bar{\Phi}_{z} \approx 0.82$. This analytic result differs from the heuristic estimates of CWL and Hlod et al. (2007), which are both equivalent to $\bar{\Phi}_{z}=0$ in our analysis. Our result $\bar{\phi}_{x} \approx 0$ has the simple physical interpretation that the thread is stable to meandering if bending forces in the heel pull the tail in the same direction as the belt motion, and unstable if the tail is instead pushed against the belt motion. This suggests a loose analogy with the difference between stable deflection of a pendulum being pulled sideways with a string and rather unstable deflection of a pendulum balanced on a pencil point pushing it sideways. The onset of meandering can thus be thought of as the heel 'losing its balance' as it pushes backwards against the belt in an attempt to slow down. Calculation of the next term (4.32) in the asymptotic expansion shows that instability occurs only if the push exceeds a small positive value.

While our aim was to determine the behaviour of the thread at neutral stability, we note that the asymptotic expansion also holds for oscillations with an $O\left(1 / \sqrt{\ln \delta_{g}}\right)$ growth rate and may thus be used to determine the growth rate of meandering oscillations close to onset.

Throughout our analysis, the effects of surface tension and inertia have been neglected for simplicity. As discussed in Appendix A, these effects can be readily accounted for provided the fall height and extrusion velocity are not too large.

We have analysed the behaviour of the thread in steady state and close to the onset of instability, but we have not considered the development of the instability into the nonlinear regime. Morris et al. (2008) presented a phenomenological fit that gives an 
estimate of the amplitude of the meanders near onset of meandering. Their analysis suggests that unsteady in-plane motion is a quadratic perturbation to the steady state and forced by the meandering state. Hence, the simple model of meandering in this paper opens up a potential avenue for analysis of in-plane perturbations and more complicated patterns.

We thank S. Morris and N. Ribe for helpful discussions, and Ribe also for providing the code used to generate the numerical results in Ribe et al. (2006b). This work was carried out with the support of an EPSRC studentship (M.J.B.).

\section{Appendix A. The effects of surface tension and inertia}

For simplicity, and partly motivated by experimental parameter values, we have thus far omitted the effects of surface tension and inertia. In the steady problem, these effects may be included by replacing (2.16) and (2.20) by

$$
\widetilde{N}_{3}=3 \pi a^{2} U^{\prime}+\pi \widetilde{\Gamma} a-\pi \widetilde{R e} U
$$

and

$$
M_{2}^{\prime}=-N_{1}-\epsilon^{2}\left(\frac{\pi a^{4}}{4} \kappa_{2} \cos \theta-\frac{\widetilde{R e} U M_{2}}{3}+\pi \widetilde{\Gamma} a^{2} \kappa_{2} a^{\prime}\right)
$$

where

$$
\widetilde{\Gamma}=\frac{\gamma}{\rho} \sqrt{\frac{\pi}{\nu g Q}} \text { and } \widetilde{R e}=\frac{g H^{3}}{v^{2}},
$$

and using $\widetilde{N}_{3}$ instead of $N_{3}$ in (2.18) and (2.19). The system of equations (2.13)-(2.20) and the boundary conditions are otherwise unchanged. The effects of surface tension and inertia on the stress-moment balance (A 2) are only $O\left(\epsilon^{2}\right)$, and therefore negligible compared with their effects on the axial stress (A 1).

We note that the dimensionless parameters $\widetilde{\Gamma}$ and $\widetilde{R e}$ are the measures of surface tension and inertia relative to viscous extensional stress that arise from the choice of the velocity scale $U_{E}$ for non-dimensionalisation. In fact, the free-fall speed $U_{F} \approx$ $0.068 U_{E}$ or the belt-speed $U_{B}$ are better representative scales for assessing the relative importance of inertia. Thus, a more appropriate condition for neglecting inertia is $R e \ll 1$ from $\S 2$ rather than $\widetilde{R e} \ll 1$.

The modification to the axial stress in (A 1) has a number of effects (which are small for $R e \ll 1$ and $\Gamma \ll 1)$. First, the solution for the tail, whether a catenary or a vertical-fall solution, is changed because the rate of stretching $U^{\prime}$ is now governed by a combination of gravity, inertia and surface tension. The new tail solutions can be found numerically by solving (3.1)-(3.5), with (3.3) replaced by (A 1). In particular, the free-fall speed $U_{f}$ is obtained by solving $\widetilde{N}_{3}^{\prime}=-\pi a^{2}$ subject to $\widetilde{N}_{3}=0$ at $s=0$ (and $U=U_{n}$ at $s=-1$ ).

Second, the changes to the tail solution mean that the horizontal force $F_{x}$ for $U_{b}>U_{f}$, the force $F_{z}$ for $U_{b}<U_{f}$, and the relationship (3.39) between $\Phi_{z}$ and $U_{b}-U_{f}$ for $U_{b} \approx U_{f}$ are all affected. However, if the new forces are used in the boundarylayer scalings (3.19) and (3.43), and the new relationship and free-fall speed are used for the transitional case of a gravitational heel, then the boundary-layer analyses in $\S \S 3.3-3.5$ are otherwise unaffected (unless $R e \gg 1$ ). The reason is that the only significant change to the system (2.13)-(2.20) is in the value of $U^{\prime}$ from (A 1) and this has negligible effect across the short length scale of the boundary layer. 
The small caveat about large $R e$ applies when the fall height is sufficiently large that most of the fall of a vertical tail occurs under an inertia-gravity balance (see Clarke 1968 for a solution). In this case, any reduction in velocity from $U_{f}$ to $U_{b}$ occurs over a compressional viscous boundary layer of height $O\left(R e^{-1 / 2}\right)$ near the belt (Dyson 2007). If $R e$ is sufficiently large, then it is no longer possible to nest the $O\left(\epsilon^{2 / 3}\right)$ bending boundary layer within this compressional boundary layer.

The third effect of inertia is on unsteady meandering oscillations. (The leadingorder effects of surface tension are again given by a simple modification to the axial stress $\widetilde{N}_{3}$.) As well as modifying the axial stress $\widetilde{N}_{3}$, inertia changes the out-of-plane stress balance (4.8) to

$$
\hat{N}_{2}=\widetilde{N}_{3} \hat{\kappa}_{1}-\bar{N}_{1} \hat{\kappa}_{3}+\hat{d}_{2 z}+\widetilde{\operatorname{Re}}\left(\hat{\omega}_{1}-\sigma \hat{U}_{y}\right)
$$

(Ribe et al. 2006b), where $\hat{U}_{y}$ is the out-of-plane velocity. The transverse inertial acceleration in (A 4) allows pendulum modes in the tail, which are analogous to the 'whirling string' modes seen in the inertio-gravitational regime of the steady coiling problem (Ribe et al. 2006b). These give rise to rather subtle effects at fall heights smaller than those at which axial inertia is significant. An analysis of these effects is currently underway and will be presented in future work.

\section{Appendix B. The effects of stretching in the tail on its horizontal deflection}

The horizontal deflection of a tail due to a horizontal force at its base was estimated in $\$ \S 3.4$ and 4.3 using the approximation that the variation of the thread radius towards the nozzle can be neglected. We now justify this approximation.

Consider a tail of unit length and possibly non-uniform radius $a$. If it hangs under its own weight and has a small horizontal force $F_{x}$ exerted on it, then the equilibrium shape is similar to the shape of a catenary. The stress equations (3.4) and (3.5) have a first integral

$$
N_{3} \sin \theta=F_{x},
$$

which can be substituted back into (3.4) to obtain

$$
\theta^{\prime}=\frac{\pi a^{2} \sin ^{2} \theta}{F_{x}} .
$$

From (3.1) and (B 2), the horizontal deflection $x_{t}$ of the tail is

$$
x_{t}=\int_{\pi / 2}^{\theta_{n}} \frac{F_{x}}{\pi a^{2} \sin \theta} \mathrm{d} \theta,
$$

where $\theta_{n}$ is the deflection of the tail from vertical at $s=-1$.

The deflection of a catenary with uniform radius $a=1$ is

$$
x_{t}=\frac{F_{x}}{\pi} \ln \left(\tan \left(\frac{\theta_{n}}{2}\right)\right),
$$

where $\theta_{n}$ is found by integration of (B 2) with $a=1$ to be

$$
\theta_{n}=\cot ^{-1}\left(\frac{\pi}{F_{x}}\right) \text {. }
$$

Hence, $x_{t}=O\left(F_{x} \ln F_{x}\right)$ as $F_{x} \rightarrow 0$. Equation (B 2) implies that $\theta=O(1)$ only in a region of $O\left(F_{x}\right)$ arclength, and hence this region gives an $O\left(F_{x}\right)$ contribution to $x_{t}$. 
Since $\theta_{n}=O\left(F_{x}\right)$, (B 3) implies that the contribution of the region $\theta=O\left(\theta_{n}\right)$ is also $O\left(F_{x}\right)$. Hence, the deflection is dominated by the region $\theta_{n} \ll \theta \ll 1$. Linearisation of $\theta$ in (B 3) and substitution of (B 5) implies that this region indeed gives the leading-order $O\left(F_{x} \ln F_{x}\right)$ contribution.

The variation of $a$ within a stretching catenary is found by considering (2.16) together with (B 1) to obtain

$$
a^{\prime}=O\left(\frac{F_{x}}{\sin \theta}\right) .
$$

Since $\theta_{n}=O\left(F_{x}\right)$ for $F_{x} \ll 1$, it follows that $a^{\prime} \ll 1$ for $\theta \gg \theta_{n}$. Hence, $a \approx 1$ for $\theta \gg \theta_{n}$ since $a=1$ at the contact point. The leading-order behaviour of the deflection is therefore not affected by the variation of thread radius in the tail.

\section{REFERENCES}

Barnes, G. \& MacKenzie, J. 1959 Height of fall versus frequency in liquid rope-coil effect. Am. J. Phys. 27, 112-115.

Barnes, G. \& Woodcock, R. 1958 Liquid rope-coil effect. Am. J. Phys. 26, 205-209.

Buckmaster, J. D., Nachman, A. \& Ting, L. 1978 The buckling and stretching of a viscida. J. Fluid Mech. 69, 1-20.

Chiu-Webster, S. \& Lister, J. R. 2006 The fall of a viscous thread onto a moving surface: a 'fluid mechanical sewing machine'. J. Fluid Mech. 569, 89-111.

Clarke, N. S. 1968 Two-dimensional flow under gravity in a jet of viscous liquid. J. Fluid Mech. 31, 481-500.

Cummings, L. J. \& Howell, P. D. 1999 On the evolution of non-axisymmetric viscous fibres with surface tension inertia and gravity. J. Fluid Mech. 389, 361-389.

Decent, S. P., King, A. C., Simmons, M. J. H, PĂrăU, E. I., Wallwork, I. M., Gurney, C. J. \& UdDin, J. 2009 The trajectory and stability of a spiralling liquid jet: viscous theory. Appl. Math. Mod. 33, 4283-4302.

Dewynne, J., Ockendon, J. R. \& Wilmott, P. 1989 On a mathematical model for fiber tapering. SIAM J. Appl. Math. 49, 983-990.

Dewynne, J. N., Howell, P. D. \& Wilmott, P. 1994 Slender viscous fibres with inertia and gravity. Q. J. Mech. Appl. Math. 47, 541-555.

Dewynne, J. N., Ockendon, J. R. \& Wilmott, P. 1992 A systematic derivation of the leading-order equations for extensional flows in slender geometries. J. Fluid Mech. 244, 323-338.

Doedel, E. J. \& Oldeman, B. E. 2009 Auto-07p: continuation and bifurcation software for ordinary differential equations. Available at: http://indy.cs.concordia.ca/auto/.

Dyson, R. J. 2007 Mathematical modelling of curtain coating. PhD thesis, University of Oxford.

Entov, V. M. \& Yarin, A. L. 1984 The dynamics of thin liquid jets in air. J. Fluid Mech. 140, 91-111.

Goren, S. L. \& Wronski, S. 1966 The shape of low-speed capillary jets of Newtonian liquids. $J$. Fluid Mech. 25, 185-198.

GriffithS, I. M. \& Howell, P. D. 2007 The surface-tension-driven evolution of a two-dimensional annular viscous tube. J. Fluid Mech. 593, 181-208.

Habibi, M., Maleki, M., Golestanian, R., Ribe, N. M. \& Bonn, D. 2006 Dynamics of liquid rope coiling. Phys. Rev. E 74 (6), 066306.

HLod, A. 2009 Curved jets of viscous fluid: interactions with a moving wall. $\mathrm{PhD}$ thesis, Eindhoven University of Technology.

Hlod, A., Aarts, A. C. T., Van De Ven, A. A. F. \& Peletier, M. A. 2007 Mathematical model of falling of a viscous jet onto a moving surface. Eur. J. Appl. Math. 18, 659-677.

Mahadevan, L., Ryu, W. S. \& Samuel, A. D. T. 1998 Fluid 'rope trick' investigated. Nature 392, 140.

Mahadevan, L., Ryu, W. S. \& Samuel, A. D. T. 2000 Correction: fluid 'rope trick' investigated. Nature 403, 502. 
Maleki, M., Habibi, M., Golestanian, R., Ribe, N. M. \& Bonn, D. 2006 Dynamics of liquid rope coiling. Phys. Rev. E 74, 066306.

Marheineke, N. \& Wegener, R. 2009 Asymptotic model for the dynamics of curved viscous fibres with surface tension. J. Fluid Mech. 622, 345-369.

Matovich, M. A. \& Pearson, J. R. A. 1969 Spinning a molten threadline: steady-state isothermal viscous flows. Ind. Engng Chem. Fundam. 8, 512-520.

Morris, S. W., Dawes, J. H. P., Ribe, N. M. \& Lister, J. R. 2008 The meandering instability of a viscous thread. Phys. Rev. E 77, 066218.

Ribe, N. M. 2004 Coiling of viscous jets. Proc. R. Soc. Lond. A 460, 3223-3239.

Ribe, N. M., Habibi, M. \& Bonn, D. $2006 a$ Stability of liquid rope coiling. Phys. Fluids 18, 084102.

Ribe, N. M., Huppert, H. E., Hallworth, M. A., Habibi, M. \& Bonn, D. $2006 b$ Multiple coexisting states of liquid rope coiling. J. Fluid Mech. 555, 275-297.

Ribe, N. M., Lister, J. R. \& ChiU-Webster, S. $2006 b$ Stability of a dragged viscous thread: onset of 'stitching' in a fluid-mechanical 'sewing machine'. Phys. Fluids 18, 124105.

TAYLOR, G. I. 1968 Instability of jets, threads and sheets of viscous fluid. In Proc. 12th Intl Cong. Appl. Math. (ed. M. Hetényi \& W. G. Vincenti), pp. 382-389. Springer.

Yarin, A. L. 1993 Free Liquid Jets and Films: Hydrodynamics and Rheology. Wiley. 